\title{
A monolithic finite-element formulation for magnetohydrodynamics
}

\author{
ARUP NANDY ${ }^{1, *(D)}$ and $\mathrm{C} \mathrm{S} \mathrm{JOG}{ }^{2}$ \\ ${ }^{1}$ Department of Mechanical Engineering, Indian Institute of Technology Guwahati, Guwahati 781039, India \\ ${ }^{2}$ Department of Mechanical Engineering, Indian Institute of Science, Bangalore, India \\ e-mail: arupn@iitg.ac.in
}

MS received 29 July 2017; revised 9 January 2018; accepted 18 January 2018; published online 27 July 2018

\begin{abstract}
This work develops a new monolithic strategy for magnetohydrodynamics based on a continuous velocity-pressure formulation. The magnetic field is interpolated in the same way as the velocity field, and the entire formulation is within a nodal finite-element framework. The velocity and pressure interpolations are chosen so that they satisfy the Babuska-Brezzi (BB) conditions. In most of the existing formulations, a stabilized formulation is used that requires a stabilization term, and some associated mesh-dependent parameters that need to be adjusted. In contrast, no such parameters need to be adjusted in the current formulation, making it more user-friendly and robust. Both transient and steady-state formulations are developed for two- and threedimensional geometries. An exact linearization of the monolithic strategy ensures that rapid (quadratic) convergence is achieved within each time (or load) step, while the stable nature of the interpolations used ensures that no instabilities arise in the solution. An existing analytical solution is corrected. The coarse mesh accuracy is shown to be better compared with other existing strategies in several benchmark problems, showing that the developed formulation is both robust and efficient.
\end{abstract}

Keywords. Incompressible MHD; monolithic; inf-sup stable.

\section{Introduction}

Magneto-hydrodynamics (MHD) is the study of flows of a conducting fluid in the presence of an electromagnetic field. Mathematically, MHD phenomena can be modelled by coupling the Navier-Stokes equations with the Maxwell equations of electrodynamics. Earlier attempts at numerical simulations of MHD flow [1-4] focused on rectangular geometries and used the finite-difference method. Some of them $[1,2]$ were not capable of capturing the boundary layer effect. A finite-element formulation for steady incompressible MHD flow under constant external magnetic field was presented in [5]. A finite-volume method was used to solve MHD problems in [6, 7]. In other works $[8,9]$, inductionless MHD phenomena have been modelled, where, instead of the Maxwell equations, only Ohm's law is used to incorporate the coupling; the magnetic induction effect cannot be modelled using this formulation.

In order to solve the coupled MHD problem, two approaches have been followed, viz., the segregated and the monolithic approaches. In the monolithic approach, both the fluid flow and the magnetic field variables are solved for simultaneously, while in the segregated approach, the Navier-Stokes equations and the Maxwell equations are solved sequentially, with the fluid flow variables obtained

*For correspondence by solving the Navier-Stokes equations passed on to the Maxwell equations to obtain the magnetic field variables, which in turn are passed back to the Navier-Stokes equations in order to solve for the fluid flow field variables, and so on. The monolithic approach converges faster, since, unlike the segregated approach, both the fluid flow and magnetic field variables are allowed to vary simultaneously within the context of, say, a Newton-Raphson strategy.

Finite-element formulations for solving the flow field in an incompressible fluid fall under two categories, viz., stable and stabilized. A stable formulation uses interpolations for pressure and velocity that satisfy the BabuskaBrezzi (BB) condition [10]. An example of a stable formulation is 'Taylor-Hood' elements, where the pressure interpolation functions are one order lower compared with those for the velocity. In contrast, in stabilized formulations, a stabilizing term is added to the variational formulation so as to circumvent the BB condition, and allow for equal-order interpolations for the velocity and pressure.

Finite-element-based strategies for solving incompressible liquid metal MHD flows have been presented in [11-23]. In [19-21, 23], edge elements have been used for discretizing the magnetic field, while nodal elements are used for modelling the fluid velocity and pressure. Most of the formulations use stabilized formulations [11-18] in order to circumvent the BB condition, and follow the segregated approach $[11-14,16]$. Although stabilized formulations 
allow the use of equal-order interpolations for the velocity and pressure, a mesh-dependent parameter needs to be set. In contrast, with a stable formulation (which is what we use in this work), there are no parameters that need to be adjusted. In $[22,23]$, higher order polynomial shape functions have been used to model the magnetostriction effect in MHD. In [22], a formulation in terms of the magnetic potential and a discontinuous pressure interpolation has been used, while in [23], a stress tensor approach has been followed in order to model the magnetostriction effect.

In the current work, we present two- and three-dimensional monolithic strategies for transient and steady-state problems that use a continuous pressure-velocity formulation (which has been shown to have better stability properties compared with a discontinuous pressure-velocity formulation in [24]) for the fluid flow variables, and an interpolation for the magnetic field that is of the same order as that of the velocity. Exact linearization of the variational formulation ensures a quadratic rate of convergence of the monolithic scheme. Comparing against analytical solutions, we show that very good coarse mesh accuracy is obtained with the proposed formulation. Because of the monolithic nature, the proposed method is efficient as well. We also propose a correction to an existing analytical solution.

\section{Mathematical formulation}

\subsection{Governing differential equations for MHD}

The strong form of the Maxwell equations is

$$
\begin{gathered}
\frac{\partial \boldsymbol{B}}{\partial t}+\nabla \times \boldsymbol{E}=\mathbf{0}, \\
\boldsymbol{\nabla} \cdot \boldsymbol{B}=0, \\
\frac{\partial \boldsymbol{D}}{\partial t}-\nabla \times \boldsymbol{H}=-\boldsymbol{j}, \\
\nabla \cdot \boldsymbol{D}=\rho_{c},
\end{gathered}
$$

where $\boldsymbol{E}$ and $\boldsymbol{H}$ are the electric and magnetic fields, respectively, $\boldsymbol{D}$ is the electric displacement (electric flux), $\boldsymbol{B}$ is the magnetic induction (magnetic flux), $\rho_{c}$ is the charge density and $\boldsymbol{j}$ is the current density. These governing equations are supplemented by the constitutive relations

$$
\begin{aligned}
& \boldsymbol{D}=\epsilon \boldsymbol{E}, \\
& \boldsymbol{B}=\mu \boldsymbol{H},
\end{aligned}
$$

where $\epsilon$ and $\mu$ are the electric permittivity and magnetic permeability, respectively. The relative permeability is defined as $\mu_{r}=\mu / \mu_{0}$, where $\mu_{0}$ is the permeability of vacuum. Substituting the constitutive relations into Eqs. (1a) and (1c), and assuming that $\epsilon$ and $\mu$ are independent of time, we get

$$
\begin{gathered}
\frac{\partial \boldsymbol{H}}{\partial t}+\frac{1}{\mu} \nabla \times \boldsymbol{E}=\mathbf{0}, \\
\epsilon \frac{\partial \boldsymbol{E}}{\partial t}-\nabla \times \boldsymbol{H}=-\boldsymbol{j} .
\end{gathered}
$$

From Eqs. (1d), (2a) and (4), we get the compatibility condition

$$
\frac{\partial \rho_{c}}{\partial t}+\nabla \cdot \boldsymbol{j}=0
$$

Under the magnetohydrodynamic assumption [12, 25], the charge relaxation time is much shorter than the transit time of electromagnetic phenomena. Hence, we assume $\partial \boldsymbol{E} / \partial t=$ 0 in Eq. (4), and $\partial \rho_{c} / \partial t=0$ in Eq. (5). Thus, the Maxwell equations reduce to

$$
\begin{gathered}
\mu \frac{\partial \boldsymbol{H}}{\partial t}+\nabla \times \boldsymbol{E}=\mathbf{0}, \\
\nabla \cdot(\mu \boldsymbol{H})=0, \\
\nabla \times \boldsymbol{H}=\boldsymbol{j}, \\
\nabla \cdot \boldsymbol{j}=0,
\end{gathered}
$$

where due to flow of the conducting fluid we have

$$
\boldsymbol{j}=\sigma(\boldsymbol{E}+\boldsymbol{u} \times \mu \boldsymbol{H}) .
$$

In this equation, $\boldsymbol{u}$ and $\sigma$ denote the fluid velocity and the conductivity of the fluid, respectively. Using Eqs. (6) and (7), we obtain the governing differential equation for $\boldsymbol{H}$ as

$$
\boldsymbol{\nabla} \times(\boldsymbol{\nabla} \times \boldsymbol{H})+\sigma \mu \frac{\partial \boldsymbol{H}}{\partial t}=\sigma \mu \nabla \times(\boldsymbol{u} \times \boldsymbol{H}) \quad \text { on } \Omega,
$$

where $\Omega$ represents the domain. On the other hand, the magnetic field exerts a body force $\mu \boldsymbol{j} \times \boldsymbol{H}$ on the fluid. Therefore, assuming an incompressible, Newtonian fluid, the governing equations for the fluid are

$$
\begin{gathered}
\boldsymbol{\nabla} \cdot \boldsymbol{u}=0 \quad \text { on } \Omega \\
\rho\left[\frac{\partial \boldsymbol{u}}{\partial t}+(\nabla \boldsymbol{u}) \boldsymbol{u}\right]=\nabla \cdot \tau+\rho \boldsymbol{b}+\mu(\nabla \times \boldsymbol{H}) \times \boldsymbol{H} \\
\boldsymbol{\tau}=-p \boldsymbol{I}+2 \mu_{v} \boldsymbol{D} \quad \text { on } \Omega \\
\boldsymbol{D}=\frac{1}{2}\left[(\nabla \boldsymbol{u})+(\nabla \boldsymbol{u})^{T}\right] \quad \text { on } \Omega \\
\boldsymbol{t}=\tau \boldsymbol{n} \quad \text { on } \Gamma \\
\boldsymbol{t}=\overline{\boldsymbol{t}} \quad \text { on } \Gamma_{t} \\
\boldsymbol{u}=\overline{\boldsymbol{u}} \quad \text { on } \Gamma_{u}
\end{gathered}
$$




$$
\boldsymbol{u}(0)=\boldsymbol{u}_{0} \quad \text { on } \Omega,
$$

where $\rho$ is the fluid density, $v$ is the kinematic viscosity, $\mu_{v}=\rho v$ is the fluid dynamic viscosity, $\boldsymbol{u}$ is the velocity, $p$ is the pressure, $\tau$ is the Cauchy stress tensor, $\boldsymbol{D}$ is the rate of deformation, $\boldsymbol{b}$ is the body force per unit mass, $\boldsymbol{t}$ is the surface traction on the boundary $\Gamma, \boldsymbol{n}$ is the outward unit normal to $\Gamma, \bar{t}$ is the prescribed traction on $\Gamma_{t}$ and $\overline{\boldsymbol{u}}$ is the prescribed velocity on $\Gamma_{u}$ with $\Gamma \equiv \Gamma_{t} \cup \Gamma_{u}$.

Summarizing, based on Eqs. (8) and (9), the coupled differential equations for magnetohydrodynamics can be written as

$$
\begin{gathered}
\boldsymbol{\nabla} \cdot \boldsymbol{u}=0 \quad \text { on } \Omega \\
\rho \frac{\partial \boldsymbol{u}}{\partial t}+\rho(\nabla \boldsymbol{u}) \boldsymbol{u}=\boldsymbol{\nabla} \cdot \tau+\rho \boldsymbol{b}+\mu(\boldsymbol{\nabla} \times \boldsymbol{H}) \times \boldsymbol{H} \\
\nabla \cdot(\mu \boldsymbol{H})=0, \\
\nabla \times(\boldsymbol{\nabla} \times \boldsymbol{H})+\sigma \mu \frac{\partial \boldsymbol{H}}{\partial t}=\sigma \mu \nabla \times(\boldsymbol{u} \times \boldsymbol{H}) \quad \text { on } \Omega
\end{gathered}
$$

\subsection{Variational formulation}

Denoting the variations of $\boldsymbol{u}$ and $\boldsymbol{p}$ by $\boldsymbol{u}_{\delta}$ and $p_{\delta}$, respectively, we can write variational statements corresponding to Eqs. (10a) and (10b) as

$$
\begin{gathered}
\int_{\Omega} p_{\delta} \boldsymbol{\nabla} \cdot \boldsymbol{u} d \Omega=0 \quad \forall p_{\delta} \\
\int_{\Omega} \rho \boldsymbol{u}_{\delta}^{T} \frac{\partial \boldsymbol{u}}{\partial t} d \Omega+\int_{\Omega} \rho \boldsymbol{u}_{\delta}^{T}(\nabla \boldsymbol{u}) \boldsymbol{u} d \Omega-\int_{\Omega}\left(\boldsymbol{\nabla} \cdot \boldsymbol{u}_{\delta}\right) p d \Omega \\
+\int_{\Omega}\left[\boldsymbol{D}_{c}\left(\boldsymbol{u}_{\delta}\right)\right]^{T} \mathbb{C}_{c} \boldsymbol{D}_{c} d \Omega=\int_{\Omega} \rho \boldsymbol{u}_{\delta}^{T} \boldsymbol{b} d \Omega \\
+\int_{\Omega} \mu \boldsymbol{u}_{\delta}^{T}[(\boldsymbol{\nabla} \times \boldsymbol{H}) \times \boldsymbol{H}] d \Omega+\int_{\Gamma_{t}} \boldsymbol{u}_{\delta}^{T} \overline{\boldsymbol{t}} d \Gamma \quad \forall \boldsymbol{u}_{\delta}
\end{gathered}
$$

where $\mathbb{C}_{c}$ is material constitutive tensor for the viscous stress, and $\boldsymbol{D}_{c}$ is rate of deformation tensor, both expressed in 'engineering' form as

$$
\boldsymbol{D}_{c}=\left[\begin{array}{c}
D_{x x} \\
D_{y y} \\
D_{z z} \\
2 D_{x y} \\
2 D_{y z} \\
2 D_{x z}
\end{array}\right], \quad \mathbb{C}_{c}=\left[\begin{array}{cccccc}
2 \mu_{v} & 0 & 0 & 0 & 0 & 0 \\
0 & 2 \mu_{v} & 0 & 0 & 0 & 0 \\
0 & 0 & 2 \mu_{v} & 0 & 0 & 0 \\
0 & 0 & 0 & \mu_{v} & 0 & 0 \\
0 & 0 & 0 & 0 & \mu_{v} & 0 \\
0 & 0 & 0 & 0 & 0 & \mu_{v}
\end{array}\right] \text {. }
$$

Note that the only nonlinear term in Eq. (11) is the $(\nabla \boldsymbol{u}) \boldsymbol{u}$ term.
Introducing a penalty term similar to the formulation in $[26,27]$ and carrying out a suitable integration by parts, the variational statement corresponding to Eq. (10d) can be written as

$$
\begin{aligned}
\int_{\Omega} \sigma \mu \boldsymbol{H}_{\delta} \cdot \frac{\partial \boldsymbol{H}}{\partial t} d \Omega+\int_{\Omega}\left(\boldsymbol{\nabla} \times \boldsymbol{H}_{\delta}\right) \cdot(\nabla \times \boldsymbol{H}) d \Omega \\
+\int_{\Omega}\left(\boldsymbol{\nabla} \cdot \boldsymbol{H}_{\delta}\right)(\boldsymbol{\nabla} \cdot \boldsymbol{H}) d \Omega-\int_{\Omega} \sigma \mu\left(\nabla \times \boldsymbol{H}_{\delta}\right) \cdot(\boldsymbol{u} \times \boldsymbol{H}) d \Omega \\
=\int_{\Gamma} \sigma \mu\left(\boldsymbol{H}_{\delta} \times \boldsymbol{n}\right) \cdot(\boldsymbol{u} \times \boldsymbol{H}) d \Gamma \\
\quad-\int_{\Gamma}\left(\boldsymbol{H}_{\delta} \times \boldsymbol{n}\right) \cdot(\boldsymbol{\nabla} \times \boldsymbol{H}) d \Gamma \quad \forall \boldsymbol{H}_{\delta},
\end{aligned}
$$

where $\boldsymbol{H}_{\delta}$ represents the variation of $\boldsymbol{H}$. Note that the penalty term satisfies Eq. (10c), which thus does not need to be considered explicitly.

Using Eqs. (6c) and (7), we can rewrite the second boundary term as $-\int_{\Gamma} \sigma\left(\boldsymbol{H}_{\delta} \times \boldsymbol{n}\right) \cdot \boldsymbol{E}$. This boundary term is zero if either $\boldsymbol{H} \times \boldsymbol{n}$ is specified or the surface is perfectly conducting $(\boldsymbol{E} \times \boldsymbol{n}=\mathbf{0})$. Thus, under these conditions, this equation reduces to

$$
\begin{aligned}
& \int_{\Omega} \sigma \mu \boldsymbol{H}_{\delta} \cdot \frac{\partial \boldsymbol{H}}{\partial t} d \Omega+\int_{\Omega}\left(\boldsymbol{\nabla} \times \boldsymbol{H}_{\delta}\right) \cdot(\boldsymbol{\nabla} \times \boldsymbol{H}) d \Omega \\
& +\int_{\Omega}\left(\boldsymbol{\nabla} \cdot \boldsymbol{H}_{\delta}\right)(\boldsymbol{\nabla} \cdot \boldsymbol{H}) d \Omega-\int_{\Omega} \sigma \mu\left(\boldsymbol{\nabla} \times \boldsymbol{H}_{\delta}\right) \cdot(\boldsymbol{u} \times \boldsymbol{H}) d \Omega \\
& \quad=\int_{\Gamma} \sigma \mu\left(\boldsymbol{H}_{\delta} \times \boldsymbol{n}\right) \cdot(\boldsymbol{u} \times \boldsymbol{H}) d \Gamma \quad \forall \boldsymbol{H}_{\delta} .
\end{aligned}
$$

We denote the field variables at times $t_{n}$ and $t_{n+1}$ by superscripts $n$ and $n+1$, respectively, and the field variables at the previous and current iterations at the current time step $t^{n+1}$ by superscripts $k$ and $k+1$ (in which case the superscript $n+1$ is suppressed). Let $t_{\Delta}^{n+1}$ denote the difference $t^{n+1}-t^{n}$. Using the generalized trapezoidal rule for the time discretization of $\boldsymbol{u}$ and $\boldsymbol{H}$, we have

$$
\begin{aligned}
\boldsymbol{u}^{n+1} & =\boldsymbol{u}^{n}+\left[(1-\alpha) \dot{\boldsymbol{u}}^{n}+\alpha \dot{\boldsymbol{u}}^{n+1}\right] t_{\Delta}^{n+1}, \\
\boldsymbol{H}^{n+1} & =\boldsymbol{H}^{n}+\left[(1-\alpha) \dot{\boldsymbol{H}}^{n}+\alpha \dot{\boldsymbol{H}}^{n+1}\right] t_{\Delta}^{n+1} .
\end{aligned}
$$

We now linearize the variational statements given by Eqs. (11) and (12) using the following incremental relations:

$$
\begin{aligned}
\boldsymbol{u}^{k+1} & =\boldsymbol{u}^{k}+\boldsymbol{u}_{\Delta}, \\
p^{k+1} & =p^{k}+p_{\Delta}, \\
\boldsymbol{H}^{k+1} & =\boldsymbol{H}^{k}+\boldsymbol{H}_{\Delta} .
\end{aligned}
$$

In modelling electromagnetic problems using nodal finite elements, one major difficulty that is encountered is the occurrence of spurious modes. A penalty term is generally added to suppress the spurious modes. Although this 
strategy works for convex domains, it fails to model sharp corners and edges. In [28], we have developed a strategy for circumventing this problem, whereby we use a thin layer of elements with zero penalty surrounding the surfaces with sharp edges, while in the rest of the domain, a nonzero penalty is used. Based on a large number of benchmark problems, we have shown in [28] that this strategy works successfully.

\subsection{Finite-element formulation}

Let the magnetic, velocity and pressure fields, and their variations (denoted by subscript $\delta$ ) and increments (denoted by subscript $\Delta$ ) be interpolated as

$$
\begin{aligned}
& \boldsymbol{H}=\boldsymbol{N} \hat{\boldsymbol{H}}, \quad \boldsymbol{u}=\boldsymbol{N} \hat{\boldsymbol{u}}, \quad p=\boldsymbol{N}_{p} \hat{\boldsymbol{p}}, \\
& \boldsymbol{H}_{\delta}=\boldsymbol{N} \hat{\boldsymbol{H}}_{\delta}, \quad \boldsymbol{u}_{\delta}=\boldsymbol{N} \hat{\boldsymbol{u}}_{\delta}, \quad p_{\delta}=\boldsymbol{N}_{p} \hat{\boldsymbol{p}}_{\delta}, \\
& \boldsymbol{H}_{\Delta}=\boldsymbol{N} \hat{\boldsymbol{H}}_{\Delta}, \quad \boldsymbol{u}_{\Delta}=\boldsymbol{N} \hat{\boldsymbol{u}}_{\Delta}, \quad p_{\Delta}=\boldsymbol{N}_{p} \hat{\boldsymbol{p}}_{\Delta} .
\end{aligned}
$$

The shape functions $\boldsymbol{N}$ for $\boldsymbol{u}$ and $\boldsymbol{H}$ are the standard Lagrange shape functions, while the pressure field interpolation $\boldsymbol{N}_{p}$ is chosen as in [24] to be continuous and one order lower ('Taylor-Hood element'), so that the mixed finite-element strategy satisfies the BB (or inf-sup) condition [10]. In this work, we use Q9/Q4/Q9 (biquadratic/bilinear/biquadratic) and B27/B8/B27 (triquadratic/trilinear/triquadratic) elements for two- and three-dimensional problems.

Using these interpolation functions, we have

$$
\begin{aligned}
\boldsymbol{D}_{c}\left(\boldsymbol{u}_{\Delta}{ }^{k+1}\right) & =\boldsymbol{B} \hat{\boldsymbol{u}}_{\Delta}^{k+1}, \\
\left(\boldsymbol{\nabla} \boldsymbol{u}_{\Delta}{ }^{k+1}\right) \boldsymbol{u}^{k} & =\boldsymbol{R} \boldsymbol{B}_{N L} \hat{\boldsymbol{u}}_{\Delta}^{k+1}, \\
\boldsymbol{\nabla} \cdot \boldsymbol{u}_{\Delta}{ }^{k+1} & =\boldsymbol{B}_{p} \hat{\boldsymbol{u}}_{\Delta}^{k+1}, \\
\nabla \times \boldsymbol{H}_{\Delta}{ }^{k+1} & =\boldsymbol{B}_{H} \hat{\boldsymbol{H}}_{\Delta}^{k+1}, \\
\nabla \cdot \boldsymbol{H}_{\Delta}{ }^{k+1} & =\boldsymbol{B}_{p} \hat{\boldsymbol{H}}_{\Delta}^{k+1},
\end{aligned}
$$

where

$$
\boldsymbol{B}=\left[\begin{array}{ccccccccc}
N_{1, x} & 0 & 0 & N_{2, x} & 0 & 0 & . & . & . \\
0 & N_{1, y} & 0 & 0 & N_{2, y} & 0 & . & . & . \\
0 & 0 & N_{1, z} & 0 & 0 & N_{2, z} & . & . & . \\
N_{1, y} & N_{1, x} & 0 & N_{2, y} & N_{2, x} & 0 & . & . & . \\
0 & N_{1, z} & N_{1, y} & 0 & N_{2, z} & N_{2, y} & . & . & . \\
N_{1, z} & 0 & N_{1, x} & N_{2, z} & 0 & N_{2, x} & . & . & .
\end{array}\right]
$$

$$
\boldsymbol{B}_{p}=\left[\begin{array}{lllllllll}
N_{1, x} & N_{1, y} & N_{1, z} & N_{2, x} & N_{2, y} & N_{2, z} & . & . & .
\end{array}\right],
$$

$$
\boldsymbol{R}=\left[\begin{array}{ccccccccc}
u_{x}^{k} & u_{y}^{k} & u_{z}^{k} & 0 & 0 & 0 & 0 & 0 & 0 \\
0 & 0 & 0 & u_{x}^{k} & u_{y}^{k} & u_{z}^{k} & 0 & 0 & 0 \\
0 & 0 & 0 & 0 & 0 & 0 & u_{x}^{k} & u_{y}^{k} & u_{z}^{k}
\end{array}\right]
$$

$$
\boldsymbol{B}_{N L}=\left[\begin{array}{ccccccccc}
N_{1, x} & 0 & 0 & N_{2, x} & 0 & 0 & . & . & . \\
N_{1, y} & 0 & 0 & N_{2, y} & 0 & 0 & . & . & . \\
N_{1, z} & 0 & 0 & N_{2, z} & 0 & 0 & . & . & . \\
0 & N_{1, x} & 0 & 0 & N_{2, x} & 0 & . & . & . \\
0 & N_{1, y} & 0 & 0 & N_{2, y} & 0 & . & . & . \\
0 & N_{1, z} & 0 & 0 & N_{2, z} & 0 & . & . & . \\
0 & 0 & N_{1, x} & 0 & 0 & N_{2, x} & . & . & . \\
0 & 0 & N_{1, y} & 0 & 0 & N_{2, y} & . & . & . \\
0 & 0 & N_{1, z} & 0 & 0 & N_{2, z} & . & . & .
\end{array}\right],
$$

$\boldsymbol{B}_{H}=\left[\begin{array}{ccccccccc}0 & -N_{1, z} & N_{1, y} & 0 & -N_{2, z} & N_{2, y} & . & . & . \\ N_{1, z} & 0 & -N_{1, x} & N_{2, z} & 0 & -N_{2, x} & . & . & . \\ -N_{1, y} & N_{1, x} & 0 & -N_{2, y} & N_{2, x} & 0 & . & . & .\end{array}\right]$.

After carrying out a linearization of the variational statements, the discretizations of the various cross-product terms that occur in this formulation are as follows:

$$
\begin{aligned}
\boldsymbol{H}_{\Delta}{ }^{k+1} \times \boldsymbol{u}^{k} & =-\boldsymbol{u}_{\mathrm{mat}}^{k} \boldsymbol{N} \hat{\boldsymbol{H}}_{\Delta}^{k+1}, \\
\boldsymbol{u}_{\Delta}{ }^{k+1} \times \boldsymbol{H}^{k} & =-\boldsymbol{H}_{\mathrm{mat}}^{k} \boldsymbol{N} \hat{\boldsymbol{u}}_{\Delta}^{k+1}, \\
\boldsymbol{u}^{k} \times \boldsymbol{H}^{k} & =-\boldsymbol{H}_{\mathrm{mat}}^{k} \boldsymbol{u}^{k}, \\
\boldsymbol{H}_{\delta} \times \boldsymbol{n} & =-\boldsymbol{n}_{\mathrm{mat}} \boldsymbol{N} \hat{\boldsymbol{H}}_{\delta}, \\
\boldsymbol{G}^{k} & =\boldsymbol{\nabla} \times \boldsymbol{H}^{k}, \\
\left(\boldsymbol{\nabla} \times \boldsymbol{H}^{k}\right) \times \boldsymbol{H}^{k} & =-\boldsymbol{H}_{\mathrm{mat}}^{k} \boldsymbol{G}^{k}, \\
\boldsymbol{u}_{\delta} \times\left(\boldsymbol{\nabla} \times \boldsymbol{H}^{k}\right) & =-\boldsymbol{G}_{\mathrm{mat}}^{k} \boldsymbol{N}_{\delta},
\end{aligned}
$$

where

$$
\begin{array}{ccr}
\boldsymbol{u}_{\mathrm{mat}}^{k}= & {\left[\begin{array}{ccc}
0 & -u_{3}^{k} & u_{2}^{k} \\
u_{3}^{k} & 0 & -u_{1}^{k} \\
-u_{2}^{k} & u_{1}^{k} & 0
\end{array}\right], \quad \boldsymbol{H}_{\mathrm{mat}}^{k}=\left[\begin{array}{ccc}
0 & -H_{3}^{k} & H_{2}^{k} \\
H_{3}^{k} & 0 & -H_{1}^{k} \\
-H_{2}^{k} & H_{1}^{k} & 0
\end{array}\right],} \\
\boldsymbol{G}_{\mathrm{mat}}^{k}=\left[\begin{array}{ccc}
0 & -G_{3}^{k} & G_{2}^{k} \\
G_{3}^{k} & 0 & -G_{1}^{k} \\
-G_{2}^{k} & G_{1}^{k} & 0
\end{array}\right], \quad \boldsymbol{n}_{\mathrm{mat}}=\left[\begin{array}{ccc}
0 & -n_{3} & n_{2} \\
n_{3} & 0 & -n_{1} \\
-n_{2} & n_{1} & 0
\end{array}\right] .
\end{array}
$$

Substituting these relations into the linearized form of the variational formulation given by Eqs. (11) and (12), we get the discrete form of the equations as

$$
\begin{aligned}
& \left(\left[\begin{array}{ccc}
\boldsymbol{M}_{u u} & \mathbf{0} & \mathbf{0} \\
\mathbf{0} & \mathbf{0} & \mathbf{0} \\
\mathbf{0} & \mathbf{0} & \boldsymbol{M}_{H H}
\end{array}\right]+\alpha t_{\Delta}^{n+1}\left[\begin{array}{ccc}
\boldsymbol{K}_{u u} & \boldsymbol{K}_{u p} & \boldsymbol{K}_{u H} \\
\boldsymbol{K}_{p u} & \mathbf{0} & \mathbf{0} \\
\boldsymbol{K}_{H u} & \mathbf{0} & \boldsymbol{K}_{H H}
\end{array}\right]\right) \\
& {\left[\begin{array}{l}
\left(\hat{\boldsymbol{u}}_{\Delta}^{k+1}\right)^{n+1} \\
\left(\hat{\boldsymbol{p}}_{\Delta}^{k+1}\right)^{n+1} \\
\left(\hat{\boldsymbol{H}}_{\Delta}^{k+1}\right)^{n+1}
\end{array}\right]=\left[\begin{array}{l}
\boldsymbol{F}_{u} \\
\boldsymbol{F}_{p} \\
\boldsymbol{F}_{H}
\end{array}\right],}
\end{aligned}
$$


where

$$
\begin{aligned}
& \boldsymbol{M}_{u u}=\int_{\Omega} \rho \boldsymbol{N}^{T} \boldsymbol{N} d \Omega \\
& \boldsymbol{M}_{H H}=\int_{\Omega} \sigma \mu \boldsymbol{N}^{T} \boldsymbol{N} d \Omega, \\
& \boldsymbol{K}_{u u}=\int_{\Omega} \rho \boldsymbol{N}^{T}\left(\nabla \boldsymbol{u}^{k}\right) \boldsymbol{N} d \Omega+\int_{\Omega} \rho \boldsymbol{N}^{T} \boldsymbol{R} \boldsymbol{B}_{N L} d \Omega \\
& +\int_{\Omega} \boldsymbol{B}^{T} \mathbb{C}_{c} \boldsymbol{B} d \Omega \\
& \boldsymbol{K}_{u p}=-\int_{\Omega} \boldsymbol{B}_{p}^{T} \boldsymbol{N}_{p} d \Omega, \\
& \boldsymbol{K}_{u H}=\int_{\Omega} \mu \boldsymbol{N}^{T} \boldsymbol{H}_{\mathrm{mat}}^{k} \boldsymbol{B}_{H} d \Omega-\int_{\Omega} \mu \boldsymbol{N}^{T} \boldsymbol{G}_{\mathrm{mat}}^{k} \boldsymbol{N} d \Omega, \\
& \boldsymbol{K}_{p u}=\int_{\Omega} \boldsymbol{N}_{p}^{T} \boldsymbol{B}_{p} d \Omega \\
& \boldsymbol{K}_{H u}=\int_{\Omega} \mu \sigma \boldsymbol{B}_{H}^{T} \boldsymbol{H}_{\mathrm{mat}}^{k} \boldsymbol{N} d \Omega-\int_{\Gamma} \mu \sigma \boldsymbol{N}^{T} \boldsymbol{n}_{\mathrm{mat}}^{T} \boldsymbol{H}_{\mathrm{mat}}^{k} \boldsymbol{N} d \Gamma, \\
& \boldsymbol{K}_{H H}=\int_{\Omega} \boldsymbol{B}_{H}^{T} \boldsymbol{B}_{H} d \Omega+\int_{\Omega} \boldsymbol{B}_{p}^{T} \boldsymbol{B}_{p} d \Omega-\int_{\Omega} \mu \sigma \boldsymbol{B}_{H}^{T} \boldsymbol{u}_{\mathrm{mat}}^{k} \boldsymbol{N} d \Omega \\
& +\int_{\Gamma} \mu \sigma \boldsymbol{N}^{T} \boldsymbol{n}_{\mathrm{mat}}^{T} \boldsymbol{u}_{\mathrm{mat}}^{k} \boldsymbol{N} d \Gamma, \\
& \boldsymbol{F}_{u}=\alpha t_{\Delta}^{n+1}\left\{\int_{\Omega} \rho \boldsymbol{N}^{T} \boldsymbol{b}^{n+1} d \Omega+\int_{\Gamma_{t}} \boldsymbol{N}^{T} \overline{\boldsymbol{t}}^{n+1} d \Gamma-\int_{\Omega} \boldsymbol{B}^{T} \tau^{k} d \Omega\right. \\
& \left.-\int_{\Omega} \rho \boldsymbol{N}^{T}\left[\left(\boldsymbol{\nabla} \boldsymbol{u}^{k}\right) \boldsymbol{u}^{k}\right] d \Omega-\int_{\Omega} \mu \boldsymbol{N}^{T} \boldsymbol{H}_{\text {mat }}^{k}\left(\boldsymbol{\nabla} \times \boldsymbol{H}^{k}\right) d \Omega\right\} \\
& +\int_{\Omega} \rho \boldsymbol{N}^{T}\left[\boldsymbol{u}^{n}-\boldsymbol{u}^{k}+(1-\alpha) t_{\Delta}^{n+1} \dot{\boldsymbol{u}}^{n}\right] d \Omega, \\
& \boldsymbol{F}_{p}=-\alpha t_{\Delta}^{n+1} \int_{\Omega} \boldsymbol{N}_{p}^{T}\left(\boldsymbol{\nabla} \cdot \boldsymbol{u}^{k}\right) d \Omega, \\
& \boldsymbol{F}_{H}=\alpha t_{\Delta}^{n+1}\left\{\int_{\Gamma} \mu \sigma \boldsymbol{N}^{T} \boldsymbol{n}_{\mathrm{mat}}^{T} \boldsymbol{H}_{\mathrm{mat}}^{k} \boldsymbol{u}^{k} d \Gamma-\int_{\Omega} \sigma \mu \boldsymbol{B}_{H}^{T} \boldsymbol{H}_{\mathrm{mat}}^{k} \boldsymbol{u}^{k} d \Omega\right. \\
& \left.-\int_{\Omega} \boldsymbol{B}_{H}^{T}\left(\nabla \times \boldsymbol{H}^{k}\right) d \Omega-\int_{\Omega} \boldsymbol{B}_{p}^{T}\left(\nabla \cdot \boldsymbol{H}^{k}\right) d \Omega\right\} \\
& +\int_{\Omega} \sigma \mu \boldsymbol{N}^{T}\left[\boldsymbol{H}^{n}-\boldsymbol{H}^{k}+(1-\alpha) t_{\Delta}^{n+1} \dot{\boldsymbol{H}}^{n}\right] d \Omega .
\end{aligned}
$$

We use a direct sparse matrix solver [29, 30] for solving the system of equations given by Eq. (14).

\subsection{Steady-state formulation}

Under steady-state conditions, the governing differential equations (10) simplify to

$$
\begin{gathered}
\boldsymbol{\nabla} \times(\boldsymbol{\nabla} \times \boldsymbol{H})=\sigma \mu \boldsymbol{\nabla} \times(\boldsymbol{u} \times \boldsymbol{H}) \quad \text { on } \Omega, \\
\boldsymbol{\nabla} \cdot \boldsymbol{u}=0 \quad \text { on } \Omega, \\
\rho(\boldsymbol{\nabla} \boldsymbol{u}) \boldsymbol{u}=\boldsymbol{\nabla} \cdot \tau+\rho \boldsymbol{b}+\mu(\boldsymbol{\nabla} \times \boldsymbol{H}) \times \boldsymbol{H} \quad \text { on } \Omega .
\end{gathered}
$$

In place of Eq. (14), we now get

$$
\left[\begin{array}{ccc}
\boldsymbol{K}_{u u} & \boldsymbol{K}_{u p} & \boldsymbol{K}_{u H} \\
\boldsymbol{K}_{p u} & \mathbf{0} & \mathbf{0} \\
\boldsymbol{K}_{H u} & \mathbf{0} & \boldsymbol{K}_{H H}
\end{array}\right]\left[\begin{array}{l}
\hat{\boldsymbol{u}}_{\Delta}^{k+1} \\
\hat{\boldsymbol{p}}_{\Delta}^{k+1} \\
\hat{\boldsymbol{H}}_{\Delta}^{k+1}
\end{array}\right]=\left[\begin{array}{c}
\boldsymbol{F}_{u} \\
\boldsymbol{F}_{p} \\
\boldsymbol{F}_{H}
\end{array}\right],
$$

where

$$
\begin{aligned}
\boldsymbol{K}_{u u}= & \int_{\Omega} \rho \boldsymbol{N}^{T}\left(\nabla \boldsymbol{u}^{k}\right) \boldsymbol{N} d \Omega+\int_{\Omega} \rho \boldsymbol{N}^{T} \boldsymbol{R} \boldsymbol{B}_{N L} d \Omega \\
& +\int_{\Omega} \boldsymbol{B}^{T} \mathbb{C}_{c} \boldsymbol{B} d \Omega \\
\boldsymbol{K}_{u p}= & -\int_{\Omega} \boldsymbol{B}_{p}^{T} \boldsymbol{N}_{p} d \Omega \\
\boldsymbol{K}_{u H}= & \int_{\Omega} \mu \boldsymbol{N}^{T} \boldsymbol{H}_{\mathrm{mat}}^{k} \boldsymbol{B}_{H} d \Omega-\int_{\Omega} \mu \boldsymbol{N}^{T} \boldsymbol{G}_{\mathrm{mat}}^{k} \boldsymbol{N} d \Omega, \\
\boldsymbol{K}_{p u}= & \int_{\Omega} \boldsymbol{N}_{p}^{T} \boldsymbol{B}_{p} d \Omega, \\
\boldsymbol{K}_{H u}= & \int_{\Omega} \mu \sigma \boldsymbol{B}_{H}^{T} \boldsymbol{H}_{\mathrm{mat}}^{k} \boldsymbol{N} d \Omega, \\
\boldsymbol{K}_{H H}= & \int_{\Omega} \boldsymbol{B}_{H}^{T} \boldsymbol{B}_{H} d \Omega+\int_{\Omega} \boldsymbol{B}_{p}^{T} \boldsymbol{B}_{p} d \Omega-\int_{\Omega} \mu \sigma \boldsymbol{B}_{H}^{T} \boldsymbol{u}_{\mathrm{mat}}^{k} \boldsymbol{N} d \Omega, \\
\boldsymbol{F}_{u}= & \int_{\Omega} \rho \boldsymbol{N}^{T} \boldsymbol{b} d \Omega+\int_{\Gamma_{t}} \boldsymbol{N}^{T} \overline{\boldsymbol{t}} d \Gamma-\int_{\Omega} \rho \boldsymbol{N}^{T}\left[\left(\nabla \boldsymbol{u}^{k}\right) \boldsymbol{u}^{k}\right] d \Omega \\
& -\int_{\Omega} \boldsymbol{B}^{T} \tau^{k} d \Omega-\int_{\Omega} \mu \boldsymbol{N}^{T} \boldsymbol{H}_{\mathrm{mat}}^{k}\left(\boldsymbol{\nabla} \times \boldsymbol{H}^{k}\right) d \Omega, \\
\boldsymbol{F}_{p}= & -\int_{\Omega} \boldsymbol{N}_{p}^{T}\left(\boldsymbol{\nabla} \cdot \boldsymbol{u}^{k}\right) d \Omega, \\
\boldsymbol{F}_{H}= & -\int_{\Omega} \sigma \mu \boldsymbol{B}_{H}^{T} \boldsymbol{H}_{\mathrm{mat}}^{k} \boldsymbol{u}^{k} d \Omega-\int_{\Omega} \boldsymbol{B}_{H}^{T}\left(\boldsymbol{\nabla} \times \boldsymbol{H}^{k}\right) d \Omega \\
& -\int_{\Omega} \boldsymbol{B}_{p}^{T}\left(\boldsymbol{\nabla} \cdot \boldsymbol{H}^{k}\right) d \Omega .
\end{aligned}
$$

These equations can be used to solve for the steady-state solution (in case it exists).

\subsection{Two-dimensional formulation}

For two-dimensional flows, we have $\boldsymbol{u}=\left(u_{x}, u_{y}\right)$ and $\boldsymbol{H}=\left(H_{x}, H_{y}\right)$. Only the $z$-component of $\boldsymbol{\nabla} \times \boldsymbol{H}$ given by $\partial H_{y} / \partial x-\partial H_{x} / \partial y$ is nonzero. In place of Eq. (13), we have

$$
\begin{aligned}
& \boldsymbol{B}=\left[\begin{array}{ccccccc}
N_{1, x} & 0 & N_{2, x} & 0 & . & . & . \\
0 & N_{1, y} & 0 & N_{2, y} & . & . & . \\
N_{1, y} & N_{1, x} & N_{2, y} & N_{2, x} & . & . & .
\end{array}\right], \\
& \boldsymbol{B}_{p}=\left[\begin{array}{lllllll}
N_{1, x} & N_{1, y} & N_{2, x} & N_{2, y} & \text {. . . . . }
\end{array}\right] \text {, } \\
& \boldsymbol{R}=\left[\begin{array}{cccc}
u_{x}^{k} & u_{y}^{k} & 0 & 0 \\
0 & 0 & u_{x}^{k} & u_{y}^{k}
\end{array}\right],
\end{aligned}
$$




$$
\begin{gathered}
\boldsymbol{B}_{N L}=\left[\begin{array}{ccccccc}
N_{1, x} & 0 & N_{2, x} & 0 & . & . & . \\
N_{1, y} & 0 & N_{2, y} & 0 & . & . & . \\
0 & N_{1, x} & 0 & N_{2, x} & . & . & . \\
0 & N_{1, y} & 0 & N_{2, y} & . & . & .
\end{array}\right], \\
\boldsymbol{B}_{H}=\left[\begin{array}{llllll}
-N_{1, y} & N_{1, x} & -N_{2, y} & N_{2, x} & . & .
\end{array}\right] .
\end{gathered}
$$

The following replacements need to be carried out in the three-dimensional formulation to obtain the two-dimensional one:

$$
\begin{aligned}
\boldsymbol{H}_{\Delta}{ }^{k+1} \times \boldsymbol{u}^{k} & \rightarrow-\left[\begin{array}{ll}
u_{x}^{k} & u_{y}^{k}
\end{array}\right] \boldsymbol{S} \hat{\boldsymbol{H}}_{\Delta}^{k+1}, \\
\boldsymbol{u}_{\Delta}{ }^{k+1} \times \boldsymbol{H}^{k} & \rightarrow-\left[\begin{array}{ll}
H_{x}^{k} & H_{y}^{k}
\end{array}\right] \boldsymbol{S} \hat{\boldsymbol{u}}_{\Delta}^{k+1}, \\
\boldsymbol{u}^{k} \times \boldsymbol{H}^{k} & \rightarrow\left[\begin{array}{ll}
u_{x}^{k} & u_{y}^{k}
\end{array}\right]\left[\begin{array}{c}
H_{y}^{k} \\
-H_{x}^{k}
\end{array}\right], \\
\left(\boldsymbol{\nabla} \times \boldsymbol{H}^{k}\right) \times \boldsymbol{H}^{k} & \rightarrow-\left(\nabla \times \boldsymbol{H}^{k}\right)_{z}\left[\begin{array}{c}
H_{y}^{k} \\
-H_{x}^{k}
\end{array}\right], \\
\boldsymbol{u}_{\delta} \times\left(\nabla \times \boldsymbol{H}^{k}\right) & \rightarrow\left(\nabla \times \boldsymbol{H}^{k}\right)_{z} \boldsymbol{S} \hat{\boldsymbol{u}}_{\delta},
\end{aligned}
$$

where

$$
\boldsymbol{S}=\left[\begin{array}{ccccccc}
0 & N_{1} & 0 & N_{2} & . & . & . \\
-N_{1} & 0 & -N_{2} & 0 & . & . & .
\end{array}\right]
$$

\section{Numerical examples}

We now demonstrate the good performance of the monolithic strategy on a number of problems. We compare the performance of the proposed method with either analytical solutions or results obtained using other numerical strategies, e.g., ones that use a stabilized formulation.

\subsection{Hartman-Poiseuille flow}

A conducting fluid flows between two parallel plates located at $y= \pm h$ under the influence of a pressure gradient $-\rho G$ and a magnetic field $\boldsymbol{H}=\left(B_{0} / \mu\right) \boldsymbol{e}_{y}$. The analytical solution is given by [31]

$$
\begin{aligned}
u_{x} & =V\left(1-\frac{\cosh (\mathrm{Ha} \eta)}{\cosh (\mathrm{Ha})}\right) \\
u_{y} & =0, \quad u_{z}=0 \\
H_{x} & =\frac{B_{0} \operatorname{Re}_{\mathrm{m}} \sinh (\mathrm{Ha} \eta)}{\mu \mathrm{Ha} \cosh (\mathrm{Ha})}-\left(1+\frac{E}{B_{0} V}\right) \frac{B_{0} \operatorname{Re}_{\mathrm{m}} \eta}{\mu}, \\
H_{y} & =\frac{B_{0}}{\mu}, \quad H_{z}=0 \\
p & =-\rho G x-\frac{\mu H_{x}^{2}}{2}
\end{aligned}
$$

where

$$
\operatorname{Re}_{\mathrm{m}}=\mu \sigma V h, \quad \mathrm{Ha}=\sqrt{\frac{\sigma}{\mu_{v}}} B_{0} h, \quad \eta=\frac{y}{h},
$$

and

$$
\begin{aligned}
& V=\frac{\rho G \mathrm{Ha}}{\sigma B_{0}^{2} \tanh (\mathrm{Ha})}, \quad E=\frac{\rho G}{\sigma B_{0}}\left(1-\frac{\mathrm{Ha}}{\tanh (\mathrm{Ha})}\right), \\
& \quad \text { for insulating walls) } \\
& V=\frac{\rho G}{\sigma B_{0}^{2}}, \quad E=0 \quad \text { (for perfectly conducting walls). }
\end{aligned}
$$

We use the same parameters as in [13], namely, $\sigma=7.14 \times$ $10^{5}(\Omega \mathrm{m})^{-1}, \quad \mu_{r}=9.2878, \quad \mu_{v}=1.5 \times 10^{-4} \mathrm{~kg} /(\mathrm{ms})$, $\rho G=4.85 \times 10^{-5} \mathrm{~Pa} / \mathrm{m}, \quad h=0.5 \mathrm{~m}, \quad B_{0}=1.4494 \times$ $10^{-4}$ Tesla (corresponding to $\mathrm{Ha}=5$ ). The domain is $[0,1] \mathrm{m} \times[-0.5,0.5] \mathrm{m}$. We impose $\boldsymbol{u}=\mathbf{0}$ and $H_{x}=0$ on the surfaces $y= \pm 0.5$, while on the surfaces $x=0,1$, we impose the traction (which in this case turns out to be the same as the pressure times the normal) obtained from the analytical solution, and $H_{y}=\mu B_{0}$. The pressure value at the origin is prescribed to be zero (datum value). We use the Q9/Q4/Q9 element for meshing; the mesh specifications for different Hartman numbers are given in table 1. Figure 1 (compare with figures 9 and 10 of [13]) shows the excellent match of the numerical and analytical results. For all cases, convergence was achieved in just 2 iterations. Nizar [12] has solved the same problem with a mesh of 1600 nodes. Shadid et al [14] use a $200 \times 200$ element mesh for $\mathrm{Ha}=20$, while in the present formulation, only a 99-node mesh suffices to get an almost perfect match with the analytical solution.

\subsection{Hartman-Poiseuille flow with external induced current}

In the Hartman-Poiseuille flow, if the duct wall has thickness $t$ and length $L$, has finite conductivity $\sigma_{p}$ and carries an external current $I$, then the solution is the same as that given by Eq. (18) except that now [31]

$$
\begin{aligned}
& E=\frac{\mathrm{Ha}}{C \mathrm{Ha}+\tanh (\mathrm{Ha})}\left(\frac{-\rho G}{\sigma B_{0}}\left[1-\frac{\tanh (\mathrm{Ha})}{\mathrm{Ha}}\right]+\frac{I}{2 \sigma L h}\right), \\
& V=\frac{\mathrm{Ha}}{C \mathrm{Ha}+\tanh (\mathrm{Ha})}\left(\frac{\rho G(1+C)}{\sigma B_{0}^{2}}-\frac{I}{2 \sigma B_{0} L h}\right),
\end{aligned}
$$

Table 1. Mesh specifications for the Hartman-Poiseuille problem.

Ha No. of elements No. of nodes No. of degrees of freedom

\begin{tabular}{lccc}
\hline 1 & $1 \times 2$ & 15 & 31 \\
2 & $1 \times 4$ & 27 & 67 \\
5 & $1 \times 8$ & 51 & 139 \\
10 & $1 \times 8$ & 51 & 139 \\
20 & $1 \times 16$ & 99 & 283 \\
\hline
\end{tabular}




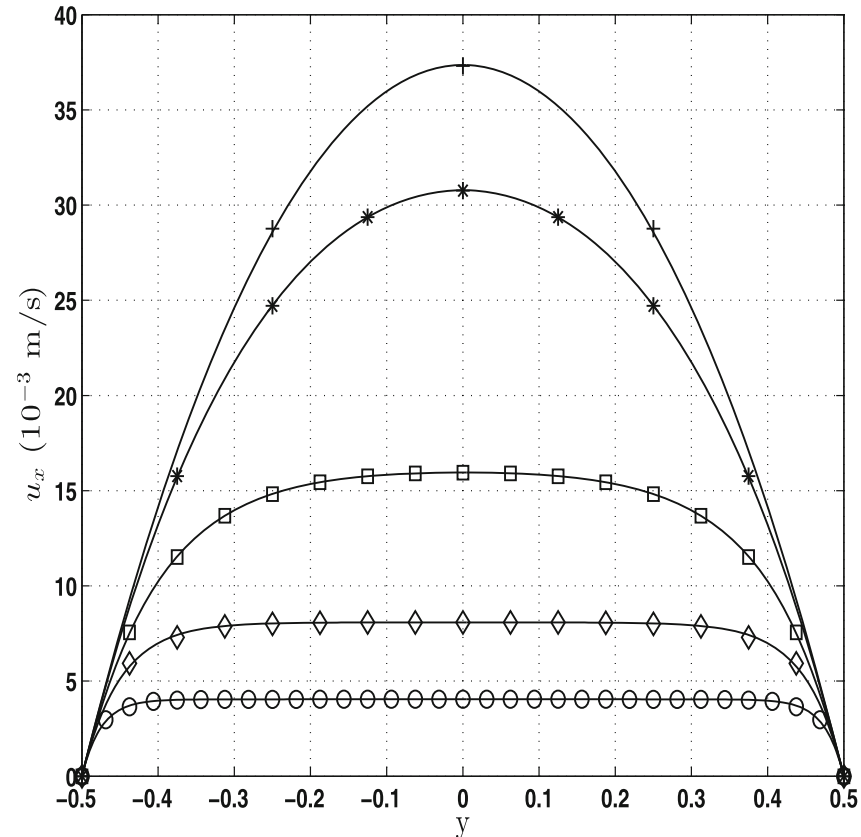

(a)

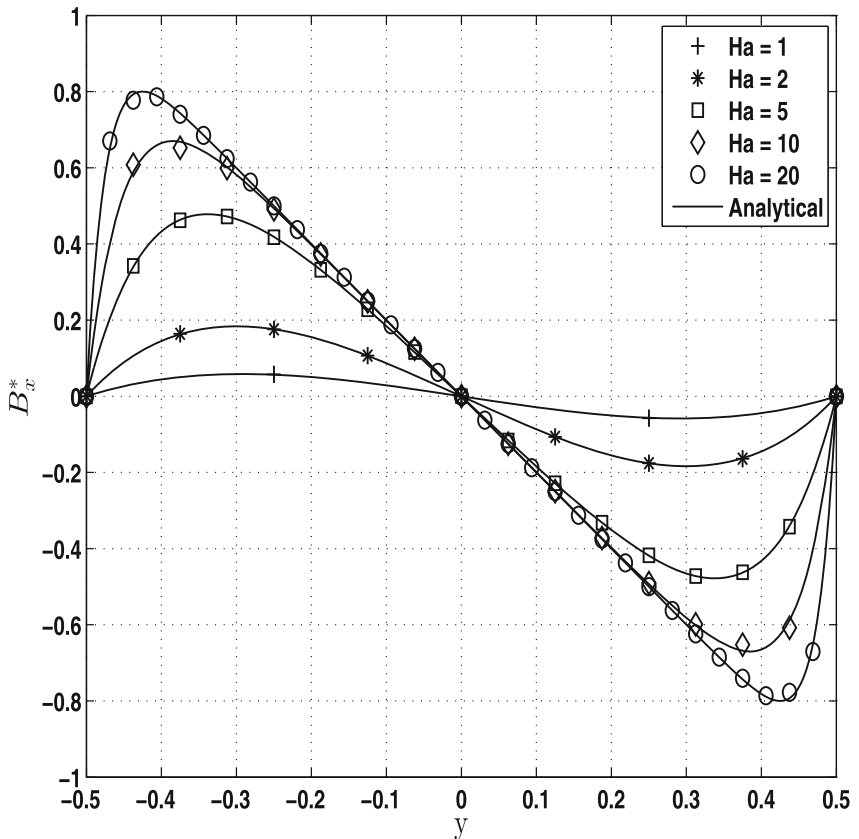

(b)

Figure 1. Variation of (a) velocity $u_{x}$ and (b) magnetic field $B_{x}^{*}=H_{x} B_{0} /(\rho G h)$ as a function of $y$ for the Hartman-Poiseuille flow with insulating boundary.

where $C=\sigma_{p} t /(\sigma h)$. For $I<0$ the system can be used as an electromagnetic pump whose discharge is greater than $I=0$. For $0<I<I_{\mathrm{cr}}$, it can be operated as an electrical generator where $I_{\mathrm{cr}}=(1+C) 2 h L \rho G / B_{0}$. For $I>I_{\mathrm{cr}}$, the Lorentz force opposes the pressure gradient, so that if it is strong enough, it can even reverse the direction of flow.

We have used the same material properties as in the insulated boundary problem. The other properties are $h=0.5 \mathrm{~m}, B_{0}=2.8988 \times 10^{-4}$ Tesla (corresponding to $\mathrm{Ha}=10), L=1 \mathrm{~m}$ and $C=0.5$. For these parameters $I_{\mathrm{cr}}=0.251$. We have used a $1 \times 8$ (51 nodes) mesh of Q9/Q4/Q9 elements for all the cases, and again convergence is obtained with two iterations for each case. Figure 2 shows the almost perfect match between the numerical and analytical values for different $I$ values. The discharge with $I<0$ is more than that with $I=0$. For $I=0.5>I_{\mathrm{cr}}$, the direction of flow reverses.

\subsection{Hartman-Couette flow}

The flow, instead of being driven by a pressure gradient as in the previous example, is driven by a prescribed velocity $u_{x}=u_{0} \quad$ on the top surface and a magnetic field $\boldsymbol{H}=\left(B_{0} / \mu\right) \boldsymbol{e}_{y}$. The bottom and top surfaces are located at $y=(0, h)$.
The analytical solution is given by [31]

$$
\begin{aligned}
u_{x}= & \frac{u_{0} \sinh (\mathrm{Ha} \eta)}{\sinh (\mathrm{Ha})}+\frac{E_{0}}{B_{0}}\left\{\frac{\sinh (\mathrm{Ha} \eta)(1-\cosh (\mathrm{Ha}))}{\sinh (\mathrm{Ha})}\right. \\
& +\cosh (\mathrm{Ha} \eta)-1\}, \\
u_{y}= & 0 \\
u_{z}= & 0, \\
H_{x}= & -\frac{B_{0} \mathrm{Re}_{\mathrm{m}} \cosh (\mathrm{Ha} \eta)}{\mu \mathrm{Ha} \sinh (\mathrm{Ha})} \\
& -\frac{\sigma E_{0} h}{\mathrm{Ha}}\left\{\frac{\cosh (\mathrm{Ha} \eta)(1-\cosh (\mathrm{Ha}))}{\sinh (\mathrm{Ha})}+\sinh (\mathrm{Ha} \eta)\right\}, \\
H_{y}= & \frac{B_{0}}{\mu}, \\
H_{z}= & 0, \\
p= & \frac{\mu}{2}\left[\left.H_{x}^{2}\right|_{\eta=0}-H_{x}^{2}\right],
\end{aligned}
$$

where

$$
\begin{aligned}
\mathrm{Re}_{\mathrm{m}} & =\mu \sigma u_{0} h, \\
\mathrm{Ha} & =\sqrt{\frac{\sigma}{\rho \nu}} B_{0} h, \\
\eta & =\frac{y}{h},
\end{aligned}
$$

and 


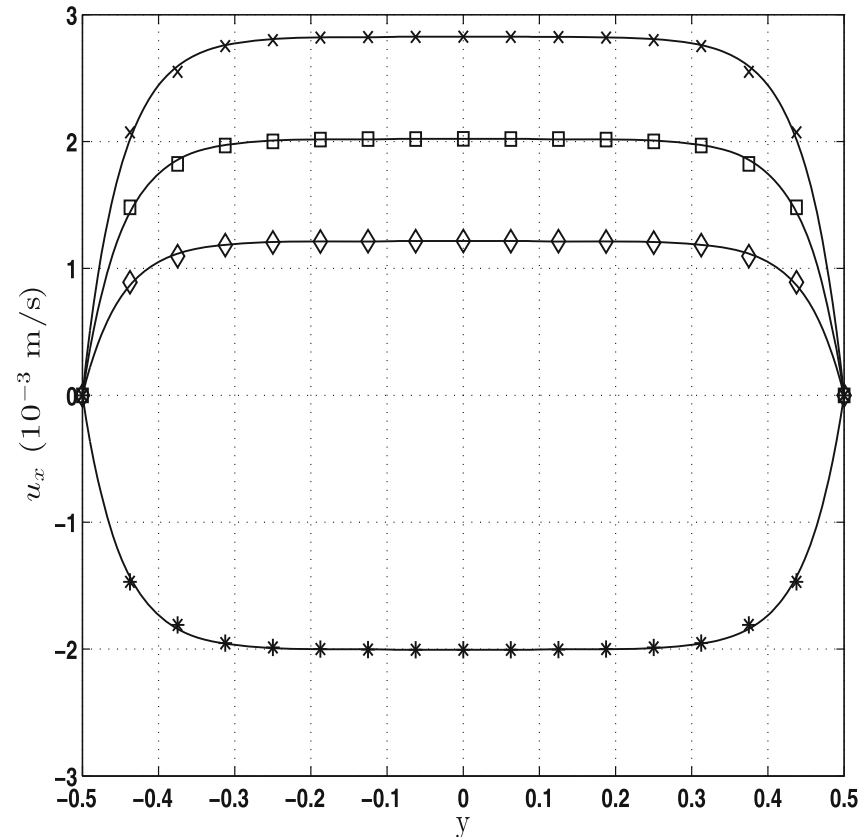

(a)

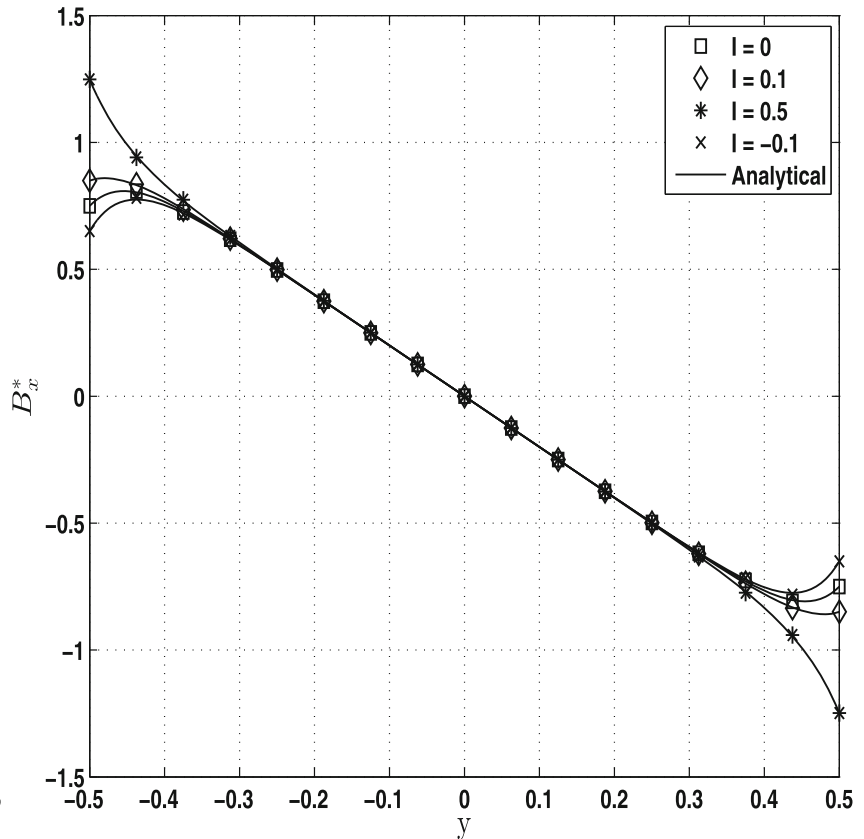

(b)

Figure 2. Variation of (a) velocity $u_{x}$ and (b) magnetic field $B_{x}^{*}=H_{x} B_{0} /(\rho G h)$ as a function of $y$ for the Hartman-Poiseuille flow with external induced current.

$$
\begin{aligned}
& E_{0}=-\frac{B_{0} u_{0}}{2}, \quad \text { (for insulating walls), } \\
& E_{0}=0, \quad \text { (for perfectly conducting walls). }
\end{aligned}
$$

The material properties are $\sigma=2(\Omega \mathrm{m})^{-1}, \mu_{r}=3.3156 \times$ $10^{6}$ and $\mu_{v}=15 \mathrm{~kg} / \mathrm{ms}$. We have chosen $B_{0}=27.3886$ and

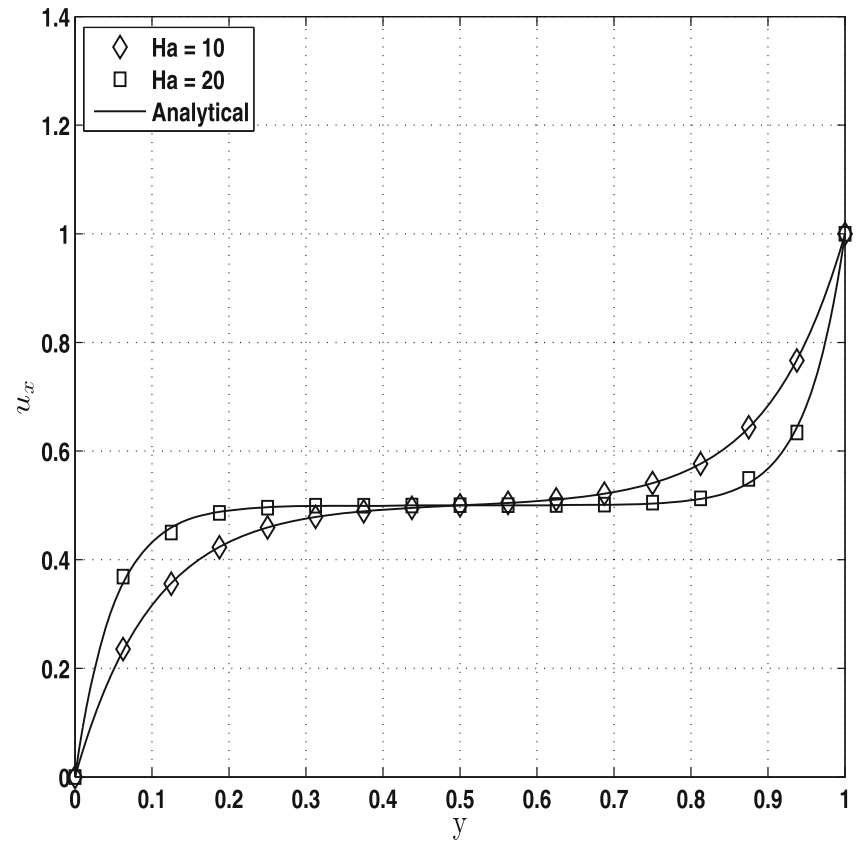

(a)
54.7722 Tesla (corresponding to $\mathrm{Ha}=10$ and 20), and $h=1 \mathrm{~m}$. The no-slip condition is imposed on the top and bottom surfaces. The traction is prescribed on the surfaces $x=0,1$, and the pressure at the origin is prescribed to zero to fix the datum value. The tangential $\boldsymbol{H}$ field is prescribed on all the boundaries.

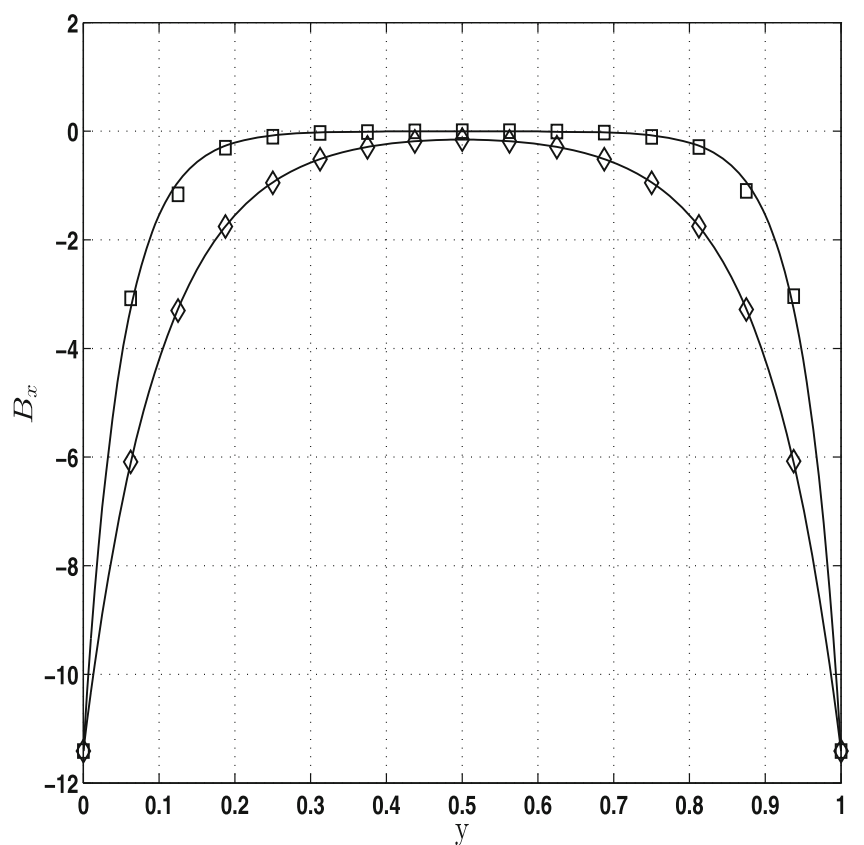

(b)

Figure 3. Variation of (a) velocity and (b) magnetic field as a function of $y$ for the Hartman-Couette flow with insulating boundary. 


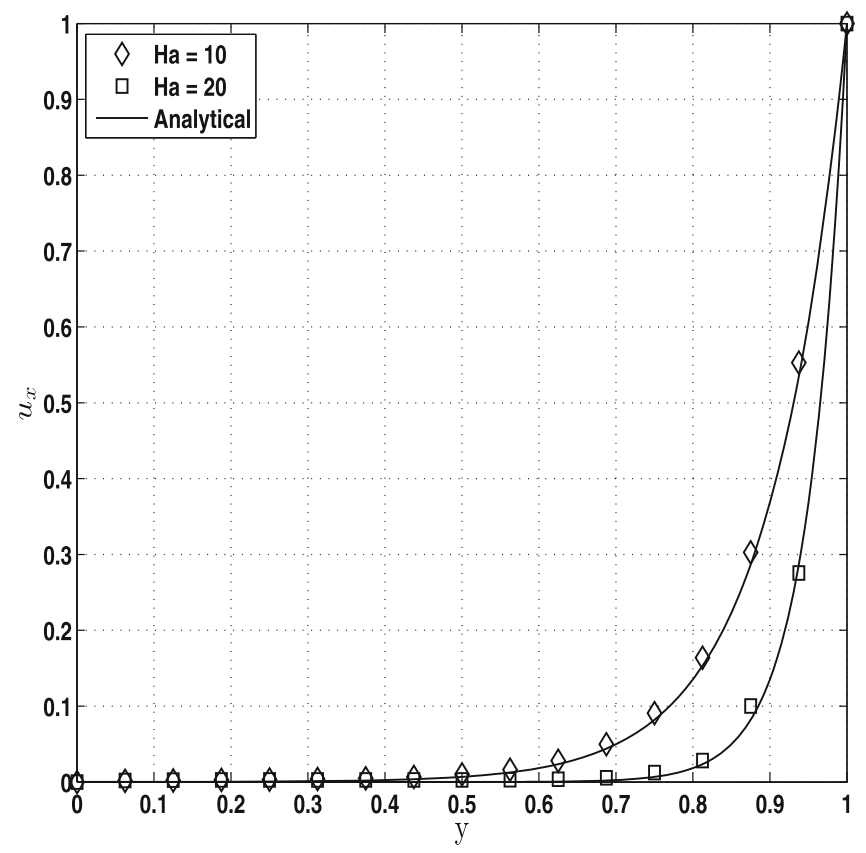

(a)

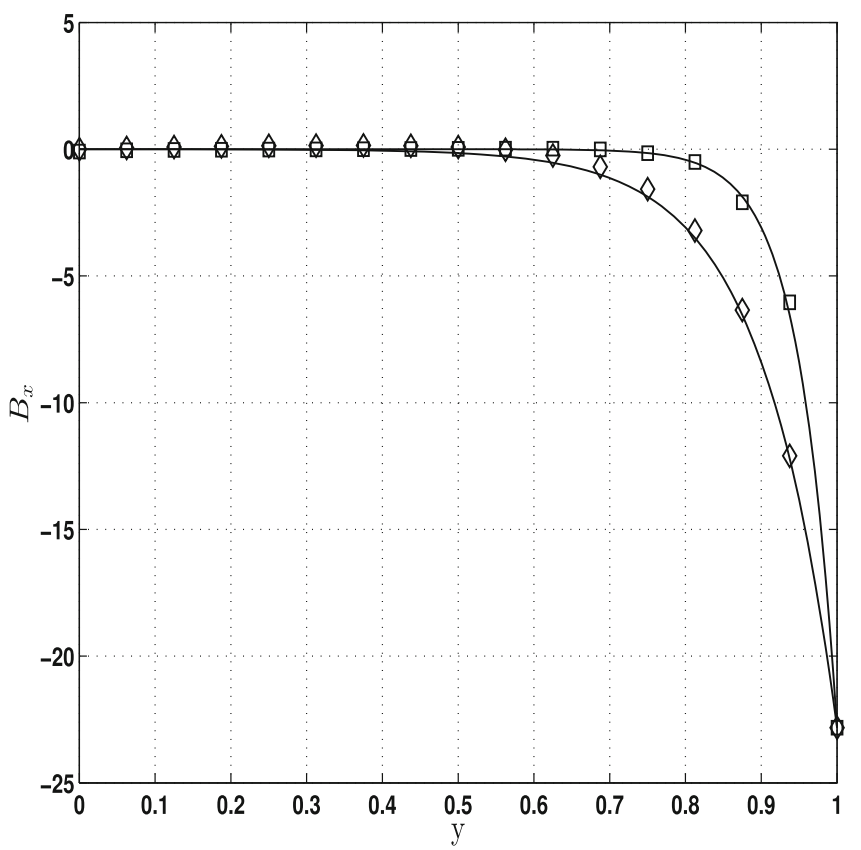

(b)

Figure 4. Variation of (a) velocity and (b) magnetic field as a function of $y$ for the Hartman-Couette flow with conducting boundary.

We have used a $1 \times 8$ mesh of Q9/Q4/Q9 elements (51 nodes and 139 degrees of freedom (dofs)) for all the cases considered. In each case, convergence is achieved in 4 iterations. Figures 3 and 4 show the almost perfect match between the numerical and analytical results for both types of boundary conditions.

\subsection{Flux expulsion problem}

An infinitely long cylinder of radius $r_{0}$ rotates with a constant angular velocity $\omega_{0}$ in a conducting medium of magnetic permeability $\mu$ and conductivity $\sigma$, in a uniform magnetic field $\left(B_{0}, 0,0\right)$, and if the flow velocity is zero outside the cylinder, then the analytical solution is given by [32], for $r \leq r_{0}$ :

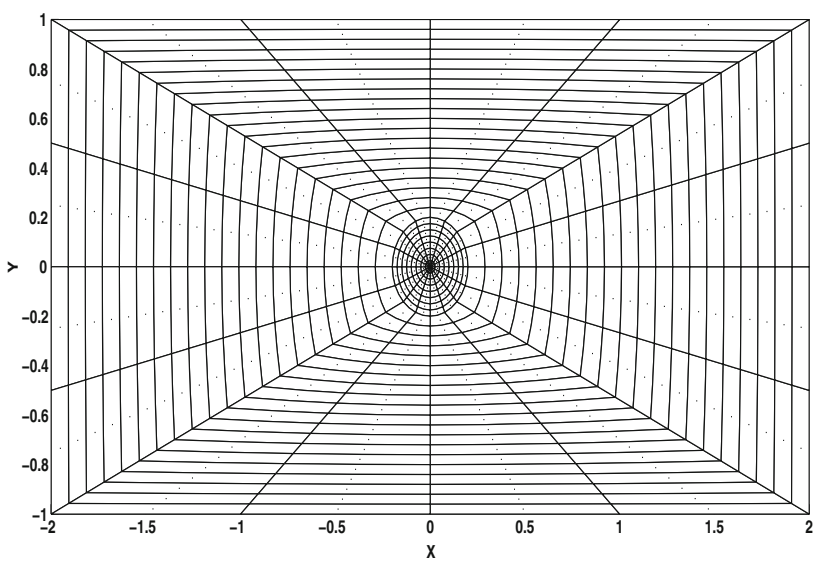

Figure 5. Mesh for the flux expulsion problem.

$$
\begin{aligned}
u_{x} & =-\omega_{0} y, \\
u_{y} & =\omega_{0} x, \\
H_{x} & =\operatorname{Im}\left[\frac{B_{0} D e^{i \theta}}{\mu}\left(\frac{q \sin \theta}{2}\left[J_{0}(q r)-J_{2}(q r)\right]+\frac{i \cos \theta J_{1}(q r)}{r}\right)\right], \\
H_{y} & =\operatorname{Im}\left[\frac{B_{0} D e^{i \theta}}{\mu}\left(\frac{i \sin \theta J_{1}(q r)}{r}-\frac{q \cos \theta}{2}\left[J_{0}(q r)-J_{2}(q r)\right]\right)\right],
\end{aligned}
$$

whereas for $r>r_{0}$

$$
\begin{aligned}
u_{x} & =0 \\
u_{y} & =0 \\
H_{x} & =\operatorname{Im}\left[\frac{B_{0} e^{i \theta}}{\mu}\left(\left[1-\frac{C}{r^{2}}\right] \sin \theta+\frac{i \cos \theta}{r}\left[\frac{C}{r}+r\right]\right)\right], \\
H_{y} & =\operatorname{Im}\left[\frac{B e^{i \theta}}{\mu}\left(\frac{i \sin \theta}{r}\left[\frac{C}{r}+r\right]-\cos \theta\left[1-\frac{C}{r^{2}}\right]\right)\right],
\end{aligned}
$$

and $u_{z}=p=H_{z}=0, \forall r$. Here, 'Im' denotes the imaginary part of the argument, and

$$
\begin{aligned}
C & =\frac{r_{0}\left[2 J_{1}\left(q r_{0}\right)-q r_{0} J_{0}\left(q r_{0}\right)\right]}{q J_{0}\left(q r_{0}\right)}, \\
D & =\frac{2}{q J_{0}\left(q r_{0}\right)}, \\
q & =\frac{(1-i) k_{0}}{\sqrt{2}}, \\
k_{0} & =\frac{\sqrt{\operatorname{Re}_{\mathrm{m}}}}{r_{0}}, \\
\mathrm{Re}_{\mathrm{m}} & =\mu \sigma \omega_{0} r_{0}^{2} .
\end{aligned}
$$




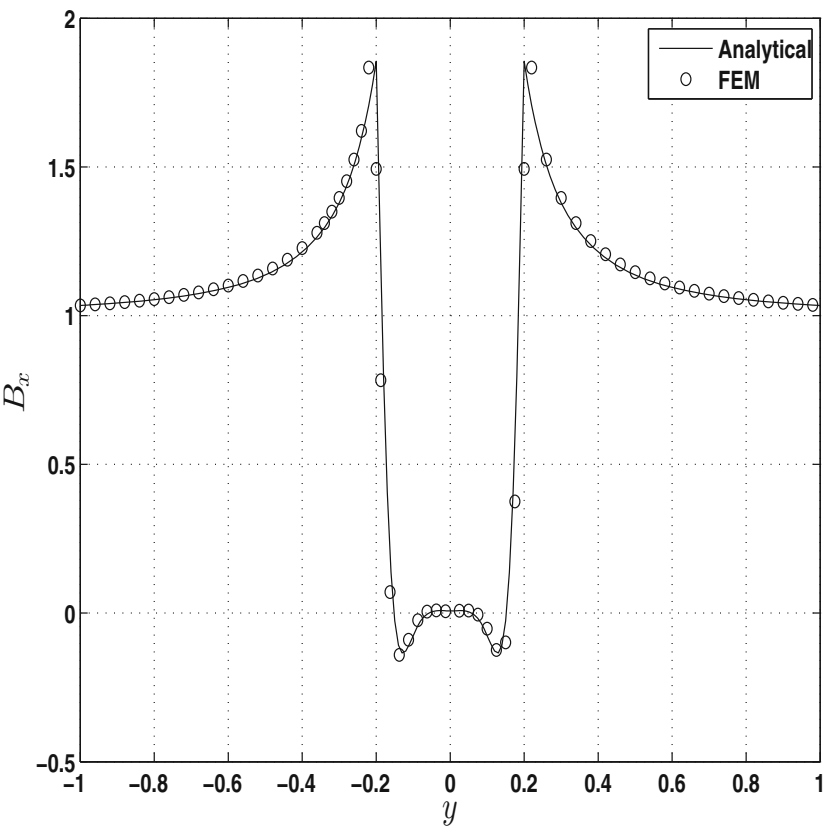

(a) $B_{x}(0, y)$

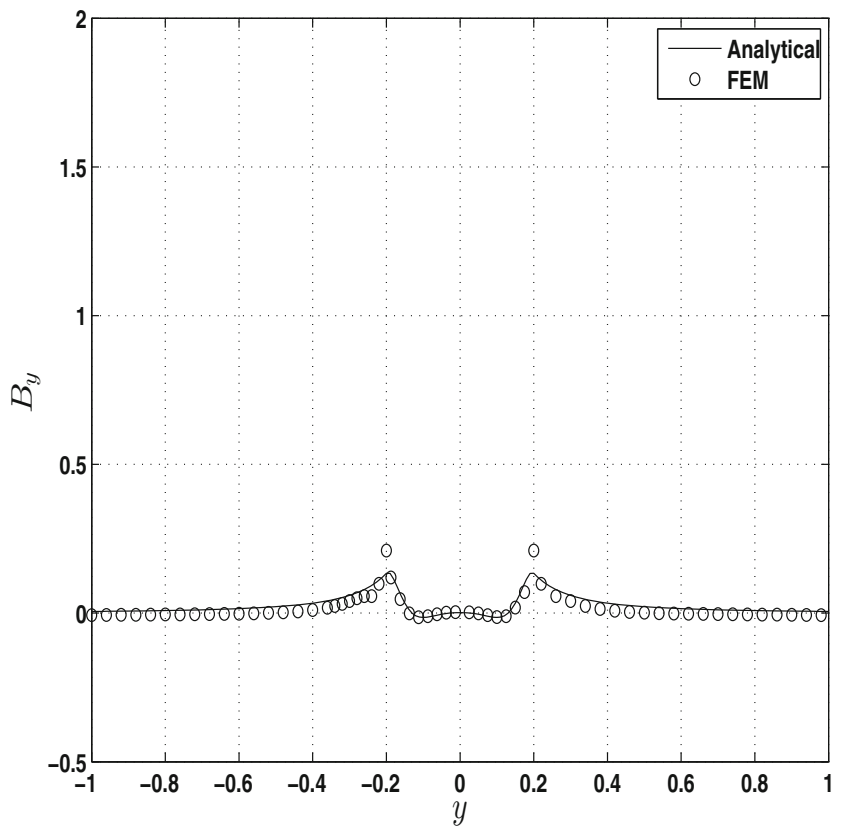

(b) $B_{y}(0, y)$

Figure 6. Variation of the magnetic field along the $y$-axis for the flux expulsion problem.

The domain used is $[-2,2] \mathrm{m} \times[-1,1] \mathrm{m}$. The values of the other parameters are $r_{0}=0.2 \mathrm{~m}, \sigma=4(\Omega \mathrm{m})^{-1}$, $\mu=0.25 \mathrm{~N} / \mathrm{A}^{2}, B_{0}=1$ Tesla and $\mathrm{Re}_{\mathrm{m}}=96$. The velocity and pressure are prescribed at all the nodes, while on the outer boundary, the tangential $\boldsymbol{H}$ field is prescribed. The used mesh of Q9/Q4/Q9 elements is shown in figure 5 (448 elements, 1777 nodes). The solution converges within 2 iterations. In [14], the same problem is solved using 20000 unstructured Q4 elements. Figure 6 shows the close match with the analytical magnetic field, both inside and outside the cylinder.

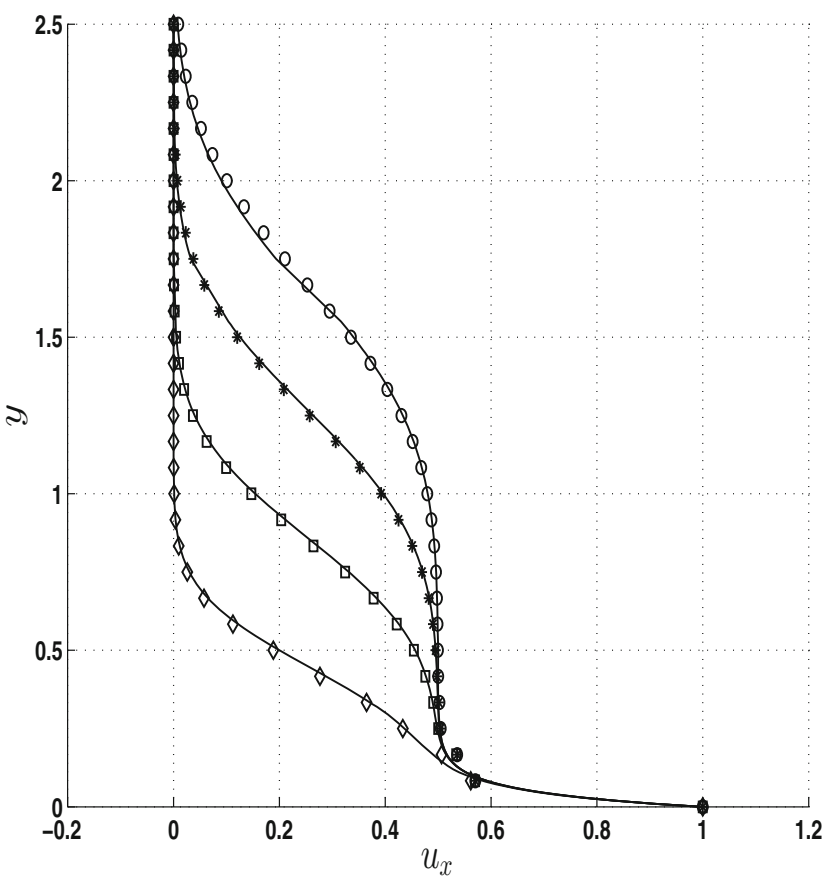

(a) $u_{x}$

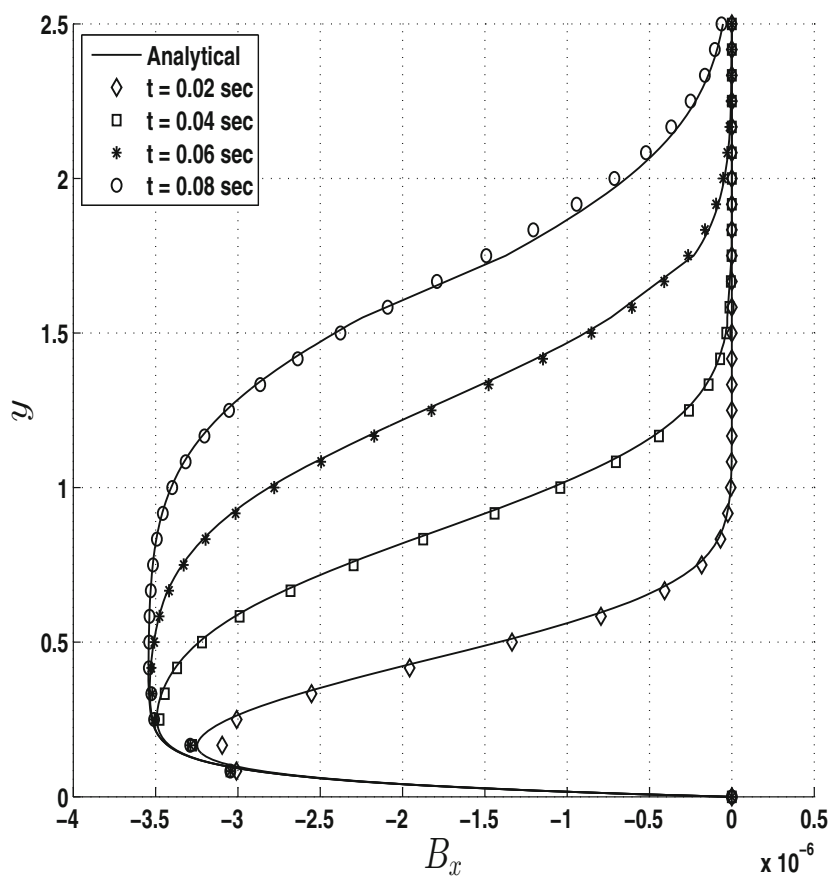

(b) $B_{x}$

Figure 7. Variation of the velocity and magnetic field along $y$ for Rayleigh flow at different times. 


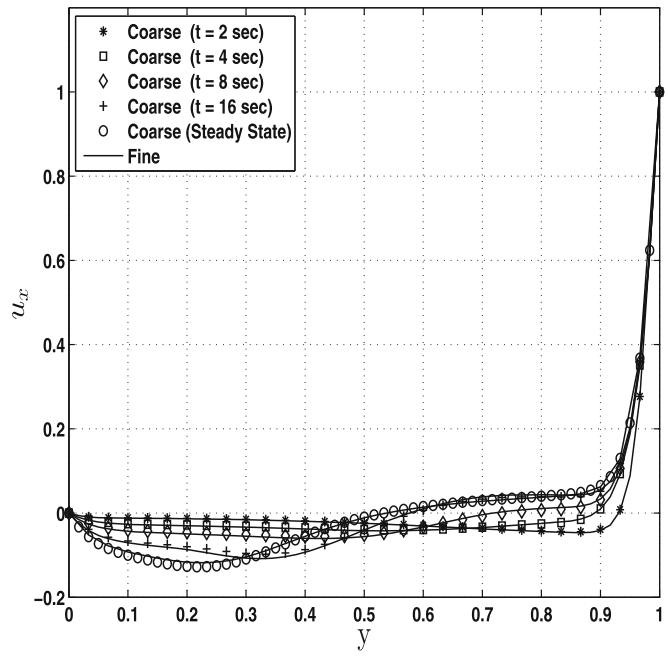

(a) $u_{x}$ as a function of $y(x=0.5 \mathrm{~m})$

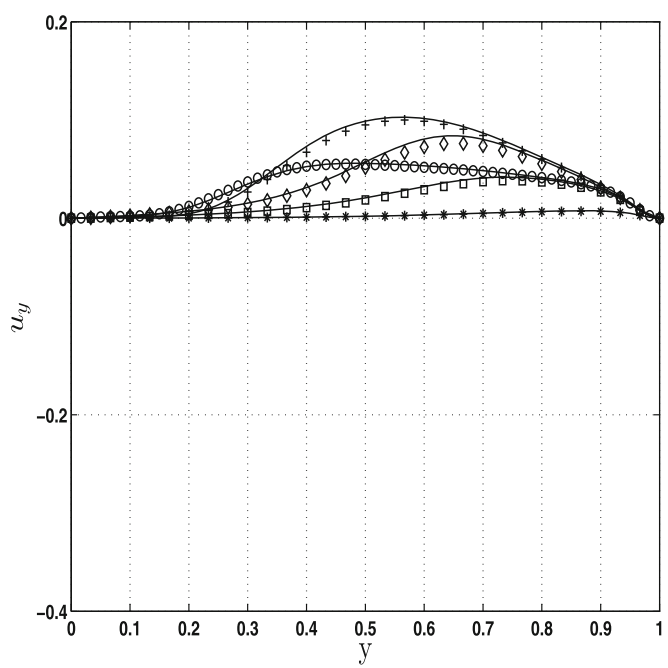

(b) $u_{y}$ as a function of $y(x=0.5 \mathrm{~m})$

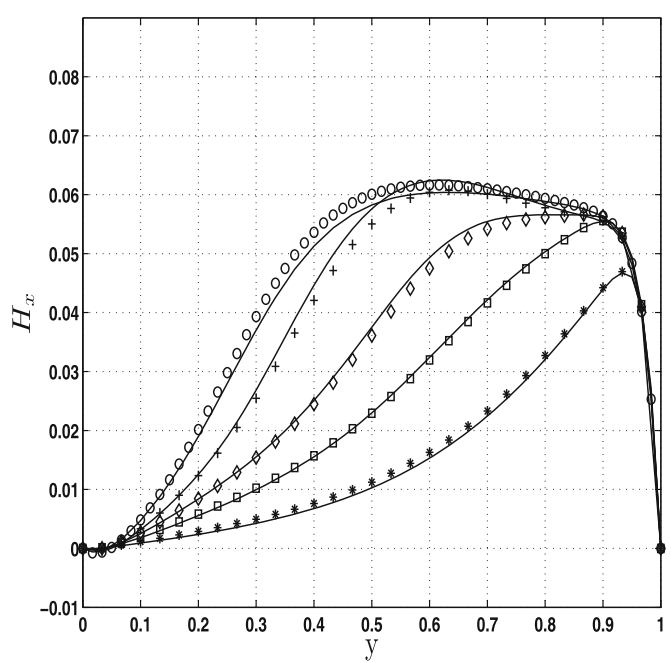

(c) $H_{x}$ as a function of $y(x=0.5 \mathrm{~m})$

Figure 8. Variation of different fields for the 2D lid-driven cavity flow.

\subsection{Rayleigh flow}

Consider a semi-infinite domain of a conducting fluid at rest, which is set in motion at $t=0$ by a constant velocity $U$ applied to the bottom surface, and a constant applied magnetic field $\boldsymbol{H}=B_{0} \boldsymbol{e}_{y}$. A diffusive wave front propagates in the direction normal to the plate. Ahead of the wavefront the fluid remains at rest, while behind the wavefront, the fluid has a nonzero velocity $u_{x}$ and induced magnetic field $H_{x}$. An analytical solution can be derived under the restriction $v=1 /(\mu \sigma)$. Denoting the error function by erf, the analytical expressions for the various field variables at time $t$ are given by [31]

$$
\begin{aligned}
u_{x}= & \frac{U}{4}\left\{2-\left[\operatorname{erf}\left(\lambda_{+}\right)+\operatorname{erf}\left(\lambda_{-}\right)\right]+e^{-A_{0} y / v}\left[1-\operatorname{erf}\left(\lambda_{-}\right)\right]\right. \\
& \left.+e^{A_{0} y / v}\left[1-\operatorname{erf}\left(\lambda_{+}\right)\right]\right\}, \\
u_{y}= & 0 \\
u_{z}= & 0, \\
H_{x}= & \frac{U \sqrt{\rho}}{4 \sqrt{\mu}}\left\{\left[\operatorname{erf}\left(\lambda_{-}\right)-\operatorname{erf}\left(\lambda_{+}\right)\right]+e^{-A_{0} y / v}\left[1-\operatorname{erf}\left(\lambda_{-}\right)\right]\right. \\
& \left.-e^{A_{0} y / v}\left[1-\operatorname{erf}\left(\lambda_{+}\right)\right]\right\}, \\
H_{y}= & \frac{B_{0}}{\mu} \\
H_{z}= & 0, \\
p= & -\frac{\mu H_{x}^{2}}{2}
\end{aligned}
$$

where

$$
\begin{aligned}
& \lambda_{ \pm}=\frac{y \pm A_{0} t}{2 \sqrt{v t}}, \\
& A_{0}=\frac{B_{0}}{\sqrt{\rho \mu}} .
\end{aligned}
$$

We have used the following values: $\rho=4 \times 10^{-5} \mathrm{~kg} / \mathrm{m}^{3}$, $\sigma=7.9577 \times 10^{5}(\Omega \mathrm{m})^{-1}, \quad \mu_{r}=1, \quad v=1 \mathrm{~m}^{2} / \mathrm{s}, \quad U=$ $1 \mathrm{~m} / \mathrm{s}$ and $B_{0}=1.4494 \times 10^{-4}$ Tesla. The domain is the rectangle $(0,1) \mathrm{m} \times(0,4) \mathrm{m}$. The same problem has also been attempted in [14].

We have used a $1 \times 24$ (147 nodes and 424 dofs) mesh of 9-node quadrilateral elements, while in [14], a mesh of $50 \times 250$ (12101 nodes) linear elements has been used. A time step of $0.001 \mathrm{~s}$ and $\alpha=0.5$ are used. The strategy converges within a maximum of 4 iterations at each time step. Figure 7 shows the almost exact match with the analytical solution (compare against figure 8 of [14]).

\section{6 $2 \mathrm{D}$ lid-driven cavity problem in the presence of a magnetic field}

The problem domain is a square with dimension $1 \mathrm{~m}$. The top surface starts moving at time $t=0$ with velocity $1 \mathrm{~m} / \mathrm{s}$. 


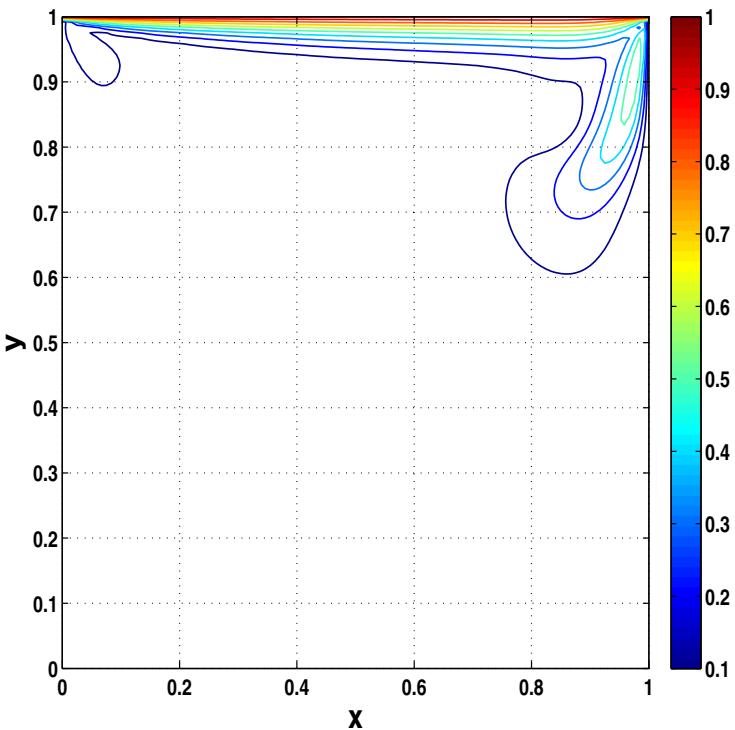

(a) $2 \mathrm{sec}$

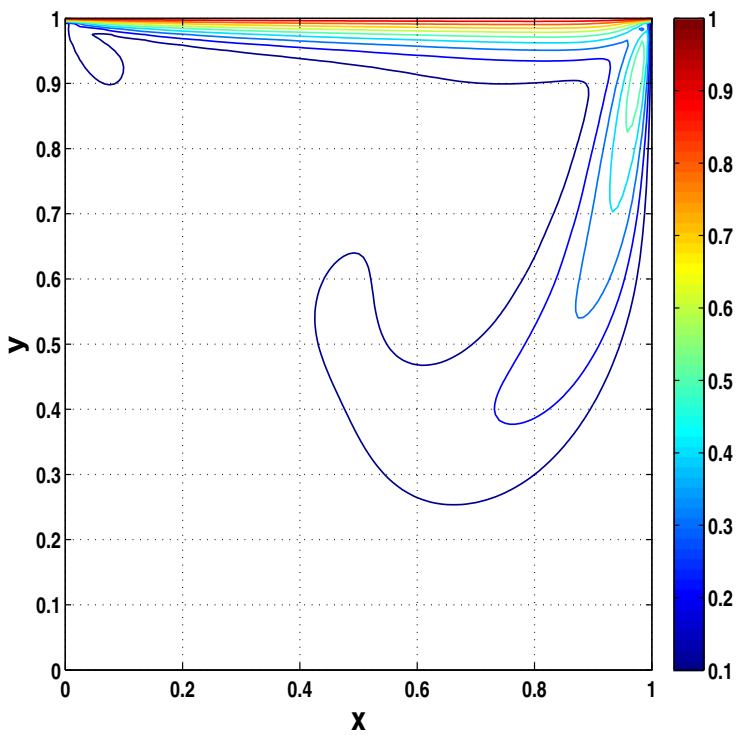

(c) $8 \mathrm{sec}$

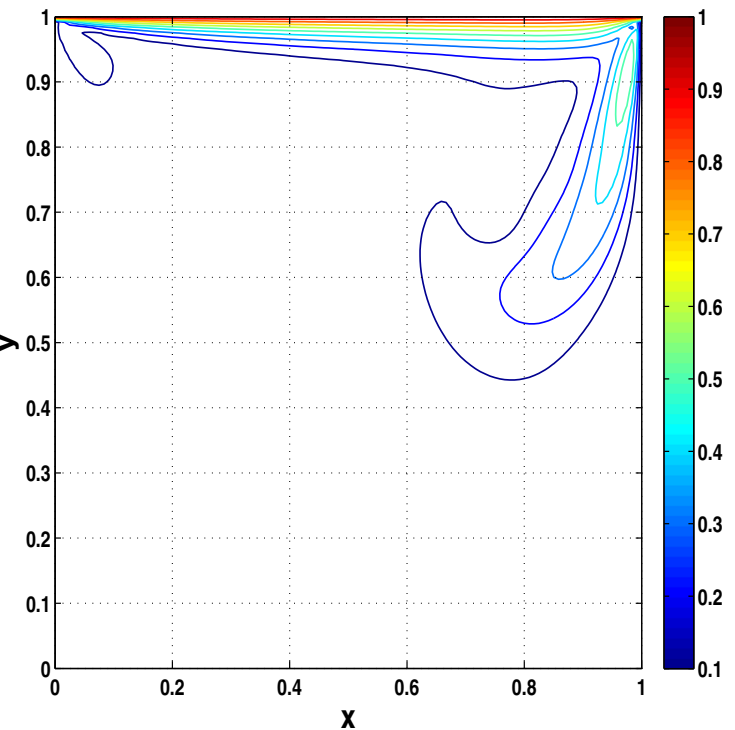

(b) $4 \mathrm{sec}$

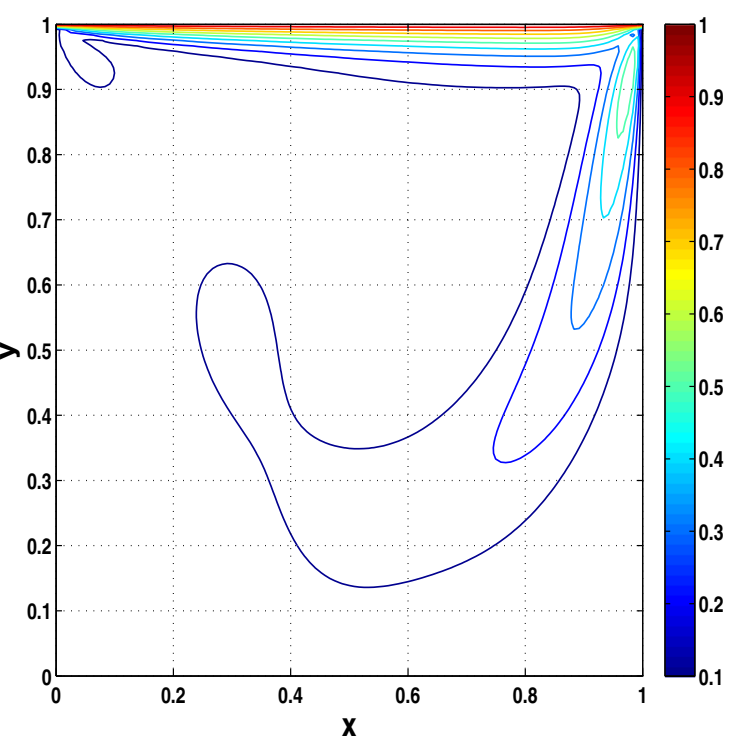

(d) $16 \mathrm{sec}$

Figure 9. Velocity contours for the 2D lid-driven cavity flow problem.

No slip boundary conditions are applied to the other three surfaces. A uniform magnetic field $\boldsymbol{H}=2 \boldsymbol{e}_{y} \mathrm{~A} / \mathrm{m}$ is applied throughout the domain at all times. Initially, $H_{x}$ is set to zero. For the top and bottom surfaces $H_{x}=0$ for all times. The conducting fluid inside the cavity has density $1000 \mathrm{~kg} / \mathrm{m}^{3}$, while the other properties are chosen so that $\mathrm{Re}=1000, \mathrm{Re}_{\mathrm{m}}=1$ and $\mathrm{Ha}=20$.

Coarse $(60 \times 60)$ and fine $(120 \times 120)$ meshes of 9 -node quadrilateral elements are used to mesh the geometry. For the steady-state analysis, 10 load steps are used, with convergence achieved within each load step within 4 iterations. For the transient analysis, we have used $\alpha=0.5$ and a time step of $0.5 \mathrm{~s}$, with convergence achieved at each time step within a maximum of 5 iterations. Figure 8 shows the evolution of the velocity profiles at various times for both levels of meshing. The convergence with respect to mesh refinement at all times is also evident from the plot. For this loading, a steady-state solution exists and is reached at sufficiently large times while performing the transient analysis. Figure 9 shows the velocity contours at different times. In order to demonstrate the quadratic convergence of our scheme, figure 10 shows the normalized error norm $\left\|\left.\boldsymbol{F}\right|_{i} /\right\| \boldsymbol{F} \|_{0}$ as a function of the iteration number at different time steps. 


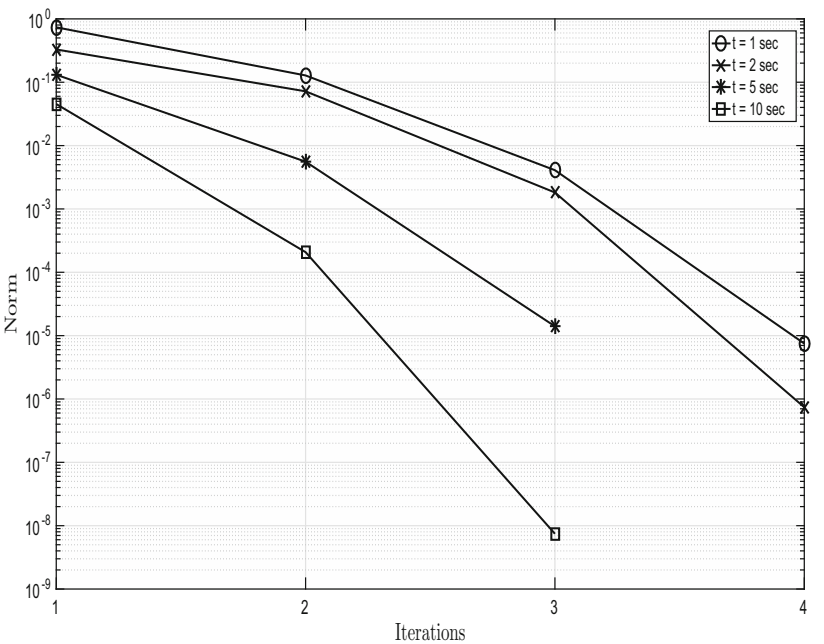

Figure 10. Convergence of the solution with iterations.

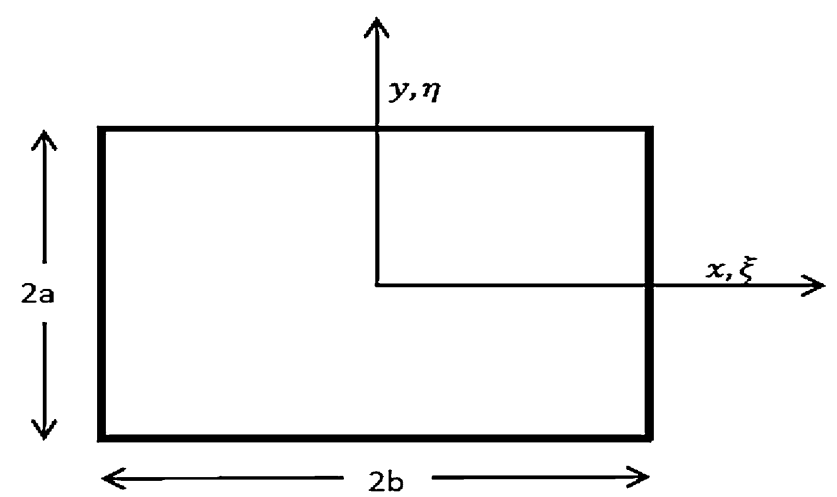

Figure 11. Hartman-Poiseuille flow through a rectangular duct.

\subsection{Hartman-Poiseuille flow through a duct with a rectangular cross-section}

A conducting fluid of density $\rho$, kinematic viscosity $v$ and conductivity $\sigma$ flows through a duct of rectangular crosssection (see figure 11). A pressure gradient $-\rho G$ is applied along the $z$-direction, and the applied magnetic field is $\boldsymbol{H}=\left(B_{0} / \mu\right) \boldsymbol{e}_{y}$. The surfaces $x= \pm b$ and $y= \pm a$ are referred to as side and Hartman wall, respectively. An analytical solution has been presented in [9, 33]. However, this analytical solution fails to satisfy the governing differential equation at $x= \pm b$ since the solution corresponding to a zero 'separation-of-variables' constant was inadvertently omitted. The corrected solution is given by

$$
\begin{aligned}
& u_{x}=0, \quad u_{y}=0, \quad u_{z}=\frac{1}{\mu_{v}} V \rho G a^{2}, \\
& H_{x}=0, \quad H_{y}=\frac{B_{0}}{\mu}, \quad H_{z}=\sqrt{\frac{\sigma}{\mu_{v}}} H \rho G a^{2}, \\
& p=-\rho G z-\frac{\mu H_{z}^{2}}{2},
\end{aligned}
$$

where, with $\xi=x / a, \eta=y / b$ and $l=b / a$,

$$
\begin{aligned}
V= & \frac{1}{2}\left(l^{2}-\xi^{2}\right)-\sum_{j=1}^{\infty} \cos \left(\frac{a_{j} \xi}{l}\right) \\
& {\left[c_{1} \cosh \left(r_{2 j} \eta\right)+c_{2} \cosh \left(r_{1 j} \eta\right)\right], } \\
H= & \sum_{j=1}^{\infty} \cos \left(\frac{a_{j} \xi}{l}\right)\left[c_{1} \sinh \left(r_{2 j} \eta\right)+c_{2} \sinh \left(r_{1 j} \eta\right)\right], \\
a_{j}= & \frac{(2 j-1) \pi}{2}, \\
r_{1 j}= & \frac{1}{2}\left(\mathrm{Ha}-s_{j}\right), \\
r_{2 j}= & \frac{1}{2}\left(\mathrm{Ha}+s_{j}\right), \\
s_{j}= & \sqrt{\mathrm{Ha}^{2}+\frac{(1-2 j)^{2} \pi^{2}}{l^{2}}} \\
\mathrm{Ha}= & \sqrt{\frac{\sigma}{\mu_{v}}} B_{0} a .
\end{aligned}
$$

The constants $c_{1}$ and $c_{2}$ depend on the boundary conditions imposed on the Hartman and side walls. The following two cases are considered.

Case Both Hartman and side walls are insulating 1

$$
c_{1}=\frac{k_{j} \sinh \left(r_{1 j}\right)}{\sinh \left(s_{j}\right)}, \quad c_{2}=-\frac{k_{j} \sinh \left(r_{2 j}\right)}{\sinh \left(s_{j}\right)}
$$

Case Conducting Hartman wall and insulating side walls 2

$$
c_{1}=-\frac{k_{j}}{2\left(1+p_{j} r_{2 j}\right) \cosh \left(r_{2 j}\right)}, \quad c_{2}=-\frac{k_{j} r_{2 j}}{s_{j} \cosh \left(r_{1 j}\right)},
$$

where

$$
\begin{aligned}
k_{j} & =\frac{16(-1)^{j} l^{2}}{(2 j-1)^{3} \pi^{3}}, \\
p_{j} & =\frac{2 \mathrm{Ha} l^{2}}{(2 j-1)^{2} \pi^{2}} .
\end{aligned}
$$

The parameters that we have used are $a=0.2 \mathrm{~m}$, $b=0.3 \mathrm{~m}, \quad \rho G=20 \mathrm{~Pa} / \mathrm{m}, \quad \sigma=100(\Omega \mathrm{m})^{-1}$, $\mu_{v}=1 \mathrm{~kg} \mathrm{~m}^{-1} \mathrm{~s}^{-1}, \rho=1 \mathrm{~kg} / \mathrm{m}^{3}, \mu=1 \mathrm{~N} / \mathrm{A}^{2}, B_{0}=5$ and 10 Tesla (corresponding to $\mathrm{Ha}=10$ and $\mathrm{Ha}=20$ ). For the simulation, we have considered the case where the side and Hartman walls are insulating. Taking into account the symmetry about the $y$-axis, we have modelled half the domain $(0,0.3) \mathrm{m} \times(-0.2,0.2) \quad \mathrm{m}$. Symmetry boundary conditions (normal $\boldsymbol{u}$ and normal $\boldsymbol{H}$ are zero) are prescribed on the boundary $x=0$. No slip boundary conditions are prescribed for the velocity, and the tangential components of $\boldsymbol{H}$ are prescribed to zero on the other three boundaries; 27-node hexahedral meshes of $5(x) \times 10(y) \times 1(z)$ and 


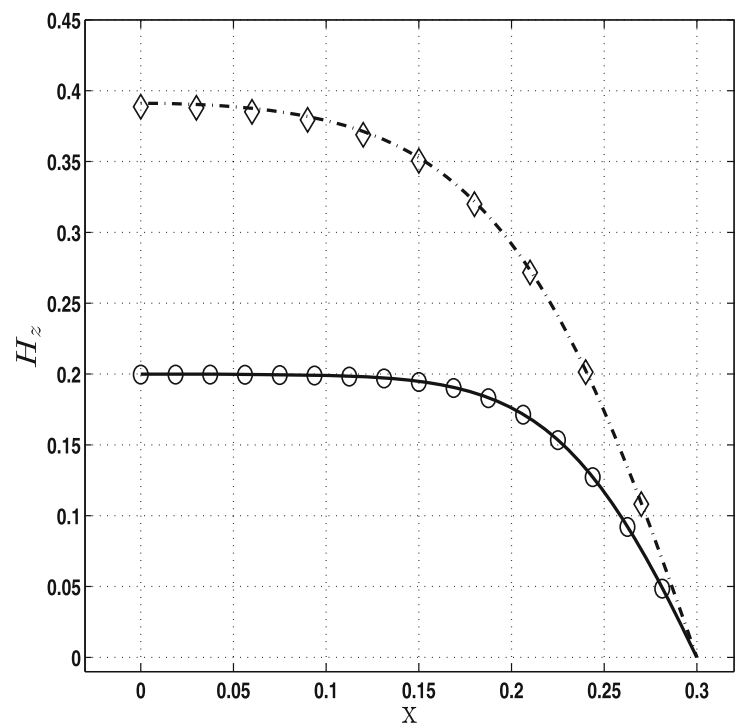

(a) $H_{z}$ along $x(y=-a / 2)$

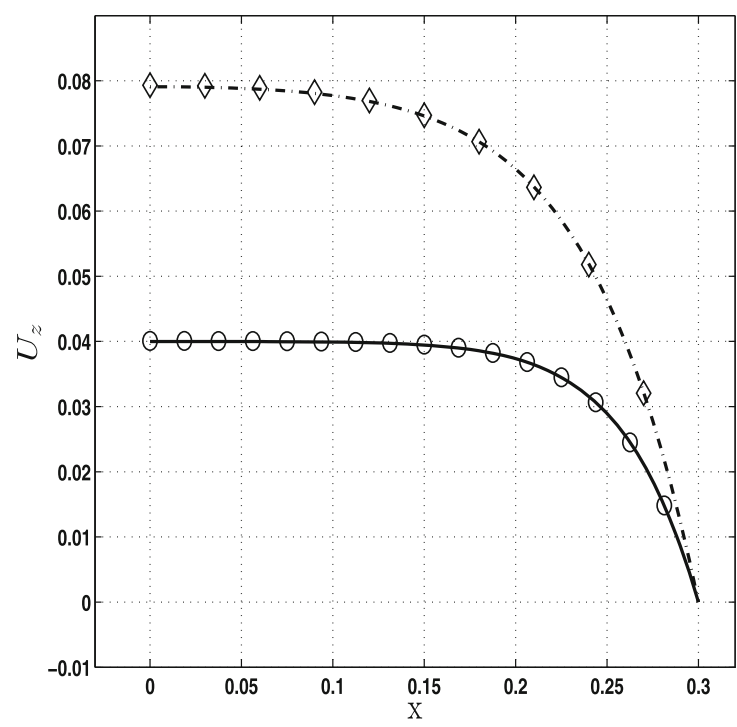

(c) $u_{z}$ along $x(y=-a / 2)$

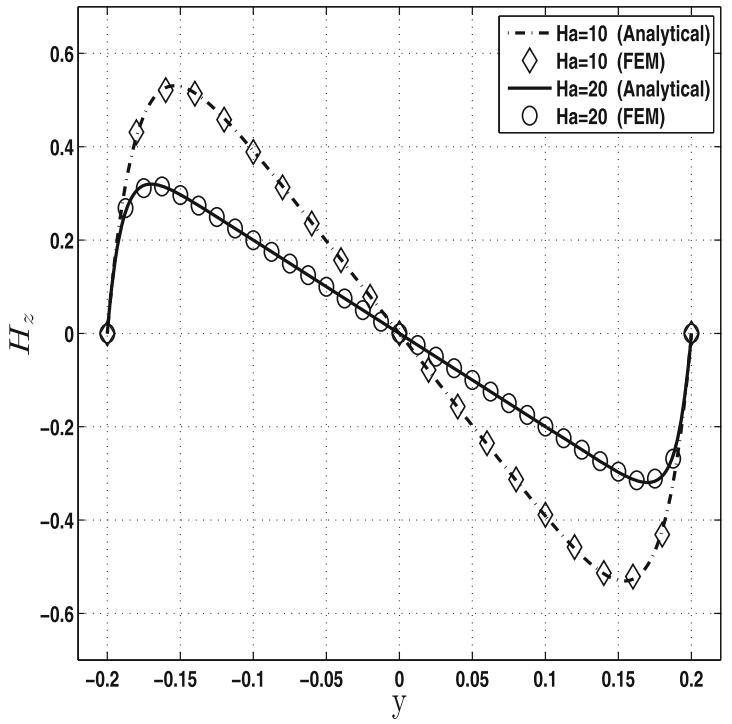

(b) $H_{z}$ along $y(x=0.0)$

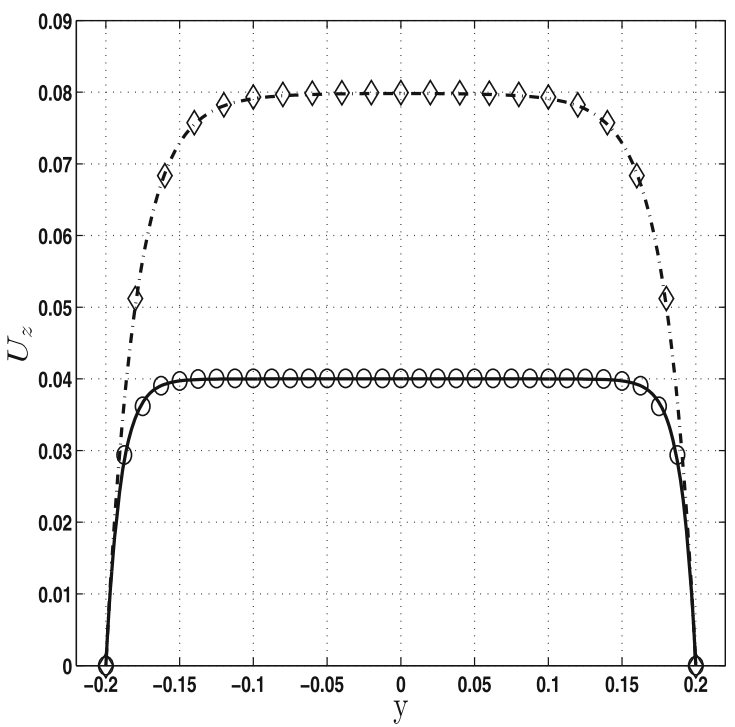

(d) $u_{z}$ along $y(x=0.0)$

Figure 12. Variation of different fields for the 3D Hartman-Poiseuille flow.

Table 2. Mesh specifications for the 3D lid-driven cavity problem.

\begin{tabular}{lccc}
\hline Mesh & $N_{b}$ & $N_{x}\left(=N_{y}\right)$ & $N_{z}$ \\
\hline Coarse & 5 & 8 & 3 \\
Fine & 8 & 10 & 3 \\
\hline
\end{tabular}

$8(x) \times 16(y) \times 1(z)$ elements for $\mathrm{Ha}=10$ and 20 , respectively, are used. We have used 10 load steps, and in each load step, convergence is achieved in a maximum of 3 iterations. Figure 12 shows the almost perfect agreement with the analytical solution for all the field variables.

\section{$3.83 D$ lid-driven cavity problem in presence of magnetic field}

Consider a cube of dimension $1 \mathrm{~m}$ filled with a conducting fluid of density $1000 \mathrm{~kg} / \mathrm{m}^{3}$. The other properties are such that $\mathrm{Re}=1000, \mathrm{Re}_{\mathrm{m}}=1$ and $\mathrm{Ha}=30$. A uniform magnetic field $\boldsymbol{H}=2 \boldsymbol{e}_{y} \mathrm{~A} / \mathrm{m}$ is applied. All other components of $\boldsymbol{H}$ and the velocity vector are initially zero. At $t=0$, the $y=1$ plane of the cavity is set in motion with velocity $u_{x}=1 \mathrm{~m} / \mathrm{s}$. The tangential magnetic fields are prescribed at the walls, i.e., for $x=0,1 \mathrm{~m}$ planes, $H_{y}=2, H_{z}=0$, for $y=0,1 \mathrm{~m}$ planes, $H_{x}=H_{z}=0$ and for $z=0,1 \mathrm{~m}$ planes, $H_{x}=0, H_{y}=2$. Because of symmetry considerations, we 


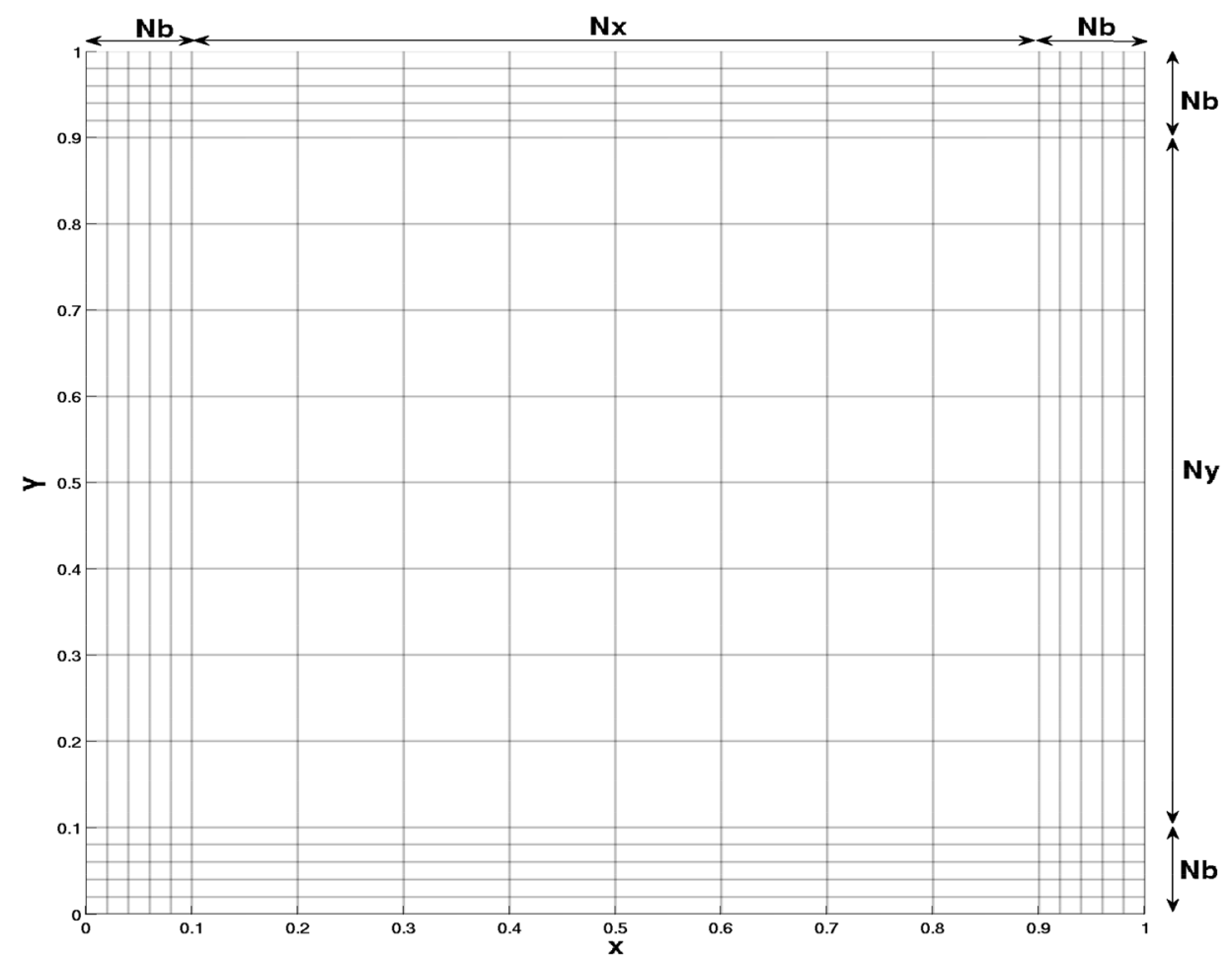

(a) $x-y$ view.

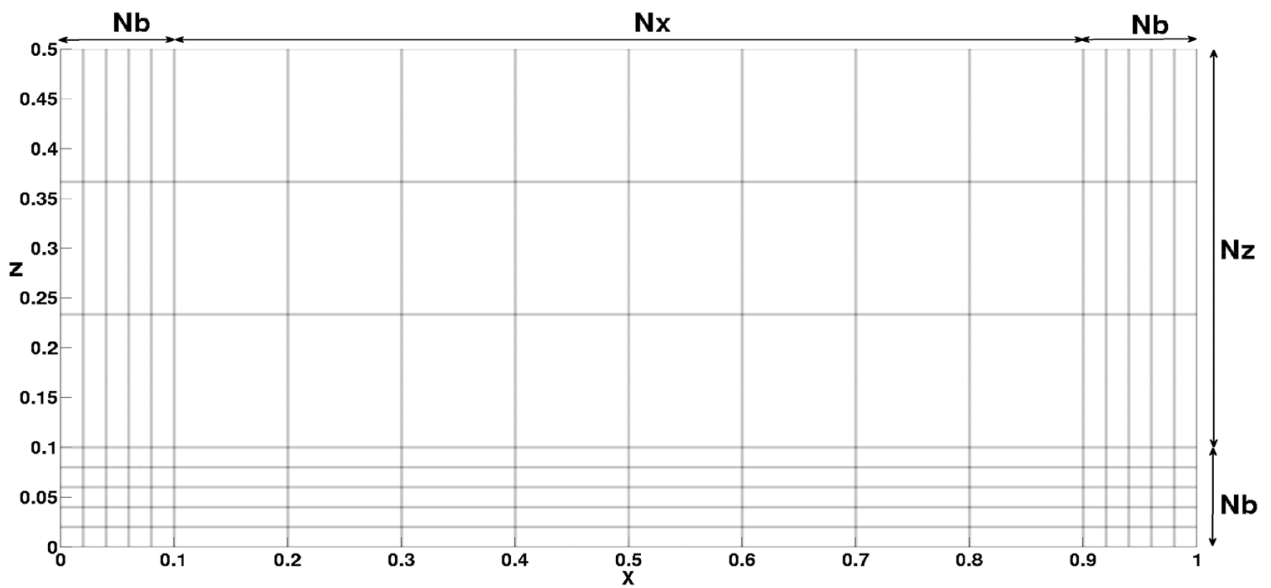

(b) $x$-z view.

Figure 13. Mesh for the 3D lid-driven cavity problem.

have modelled half the domain given by $(0,1) \mathrm{m} \times(0,1) \mathrm{m} \times(0,0.5) \mathrm{m}$. The boundary conditions $u_{z}=0$ and $H_{z}=0$ are prescribed on the symmetry face.

A graded mesh of 27-node hexahedral elements (with a finer mesh near the walls) is used to model this problem. Table 2 presents the mesh specifications for the coarse and fine meshes with $N_{b}$ denoting the number of elements in the $0.1 \mathrm{~m}$ region from each of the 5 boundaries (other than the symmetry boundary), $N_{x}$ and $N_{y}$ denoting the number of elements along the $x$ - and $y$-directions, respectively, for the intermediate $0.8 \mathrm{~m}$ region, and $N_{z}$ denoting the number of elements in the top $0.4 \mathrm{~m}$ region along the $z$-direction. Figure 13 shows the $x-y$ and $x-z$ views of the coarse mesh.

The time step is taken to be $0.5 \mathrm{~s}$. Convergence is achieved within a maximum of 5 iterations in each time step. The steady-state analysis is performed using 10 load steps, and convergence is achieved in a maximum of 4 iterations at each load step. For $\mathrm{Re}=1000$ and $\mathrm{Ha}=20$, figure 14 presents the evolution of different field variables along $x=0.5$ and $y=0.5$ on the mid $z$-plane as a function of time. Convergence of the solution with respect to mesh refinement is evident from the plot. At higher times (not 


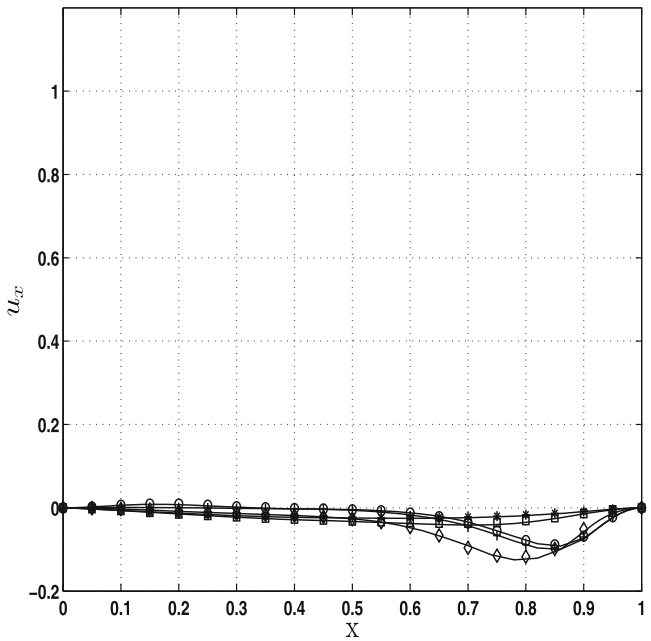

(a) $u_{x}$ along $x(y=0.5 \mathrm{~m}, z=0.5 \mathrm{~m})$

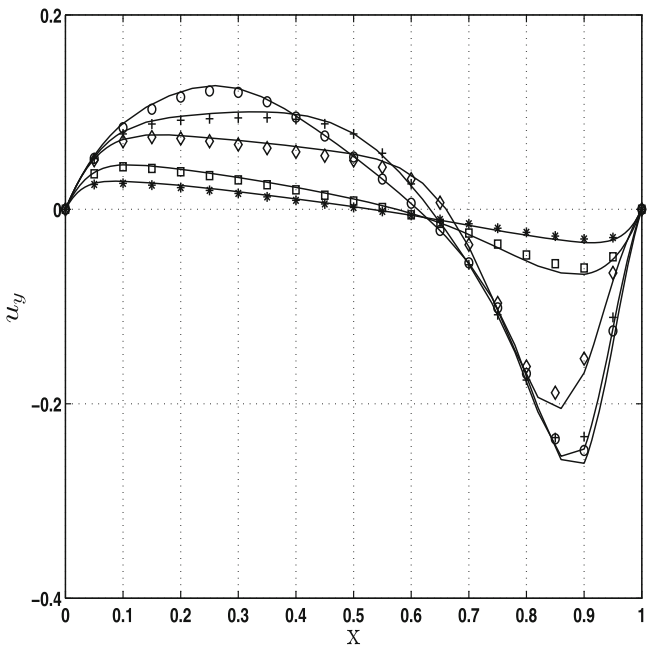

(c) $u_{y}$ along $x(y=0.5 \mathrm{~m}, z=0.5 \mathrm{~m})$

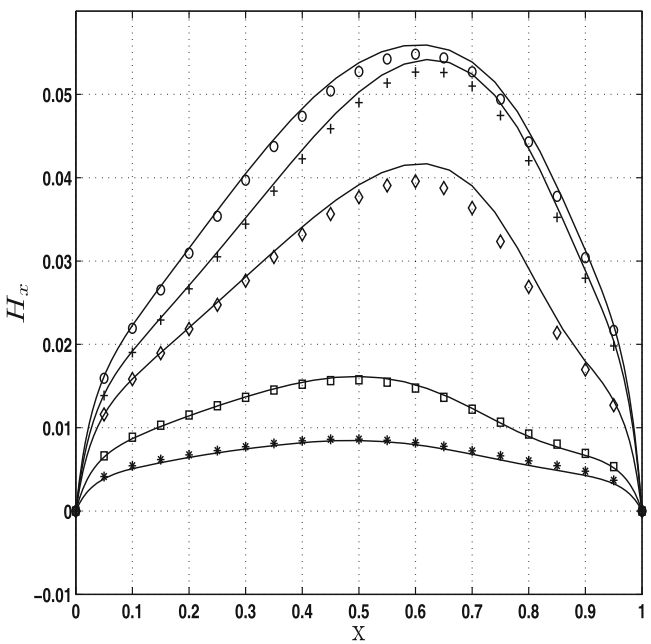

(e) $H_{x}$ along $x(y=0.5 \mathrm{~m}, z=0.5 \mathrm{~m})$

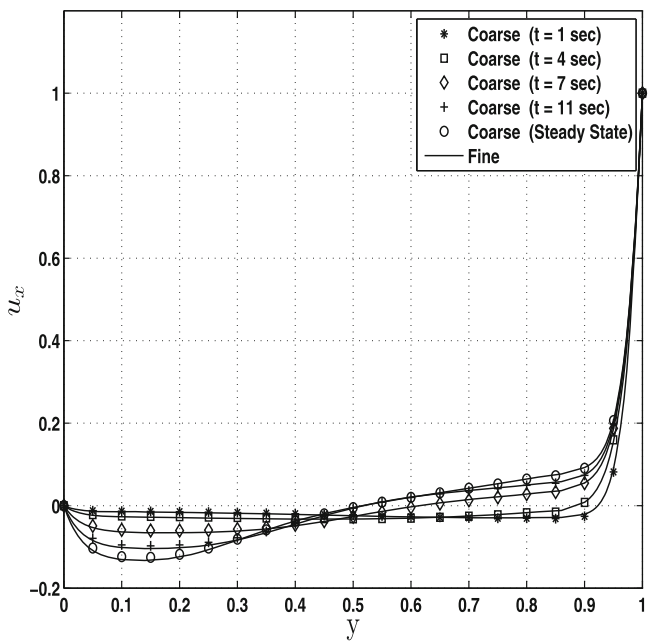

(b) $u_{x}$ along $y(x=0.5 \mathrm{~m}, z=0.5 \mathrm{~m})$

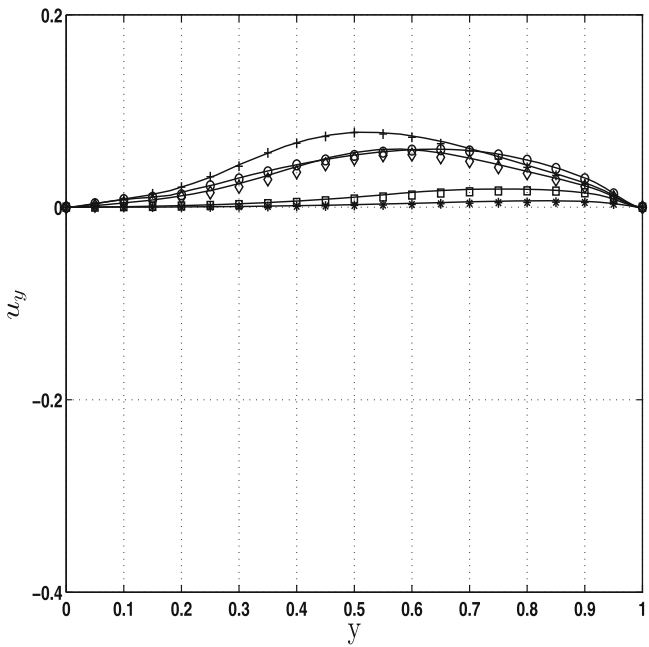

(d) $u_{y}$ along $y(x=0.5 \mathrm{~m}, z=0.5 \mathrm{~m})$

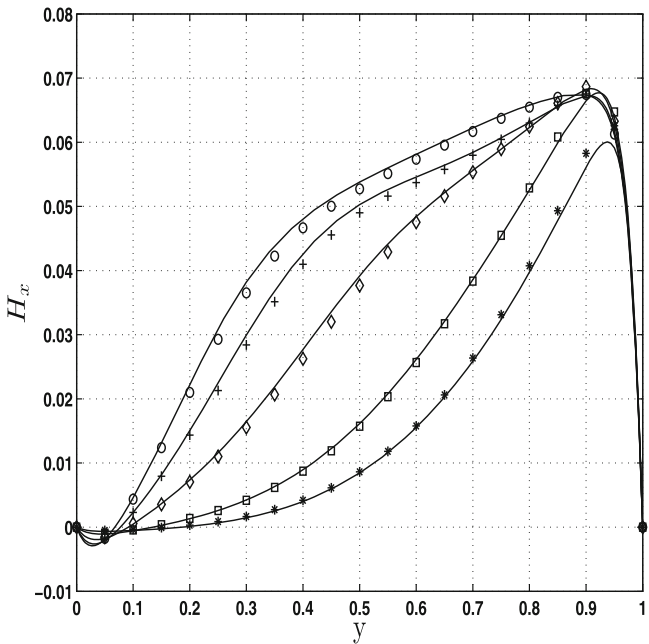

(f) $H_{x}$ along $y(x=0.5 \mathrm{~m}, z=0.5 \mathrm{~m})$

Figure 14. Variation of the different fields with time on the $z=0.5 \mathrm{~m}$ plane in the $3 \mathrm{D}$ lid-driven cavity problem $(\operatorname{Re}=1000, \mathrm{Ha}=20)$. 
shown in the plot), all field variables converge to the steady-state solution.

\section{Conclusion}

In this work, a monolithic strategy for magnetohydrodynamics based on a continuous pressure interpolation (which is known to be BB stable) has been presented. Both fully transient and steady-state formulations for two- and threedimensional problems have been developed. The exact linearization used in the monolithic strategy ensures rapid convergence within each time (or load) step, while the BBstable nature of the interpolations used ensures that no instabilities arise in the solution. An existing analytical solution has been corrected, and a new analytical solution for wedge-type geometries has been presented. A variety of steady-state and transient problems have been solved and compared in many cases against analytical solutions or against existing numerical solutions. The developed strategy is shown to yield extremely good coarse-mesh accuracy and is, thus, both robust and efficient.

\section{Nomenclature}

$\alpha$

b

B

$\mathbb{C}$

D

$\boldsymbol{D}_{c}$

E

$\epsilon$

$\epsilon_{0}, \epsilon_{r}$

$\Gamma$

$\mathrm{Ha}$

$\boldsymbol{H}$

$j$

$\mu$

$\mu_{0}, \mu_{r}$

$\mu_{v}$

n

$v$

$\Omega$

$p$

$\mathrm{Re}$

$\mathrm{Re}_{\mathrm{m}}$

$\rho$

$\rho_{c}$

$\sigma$

$t$

$t$

$t_{\Delta}$

$\tau$

$\boldsymbol{u}$ parameter in the generalized trapezoidal rule body force per unit mass

magnetic induction

material constitutive tensor

electric displacement

rate of deformation tensor

electric field

electric permittivity

$\epsilon$ of vacuum, relative $\epsilon$

boundary

Hartman number

magnetic field

current density

magnetic permeability

$\mu$ of vacuum, relative $\mu$

fluid dynamic viscosity

unit normal vector

kinematic viscosity

domain

fluid pressure

Reynolds number

magnetic Reynolds number

fluid density

charge density

conductivity

time

traction vector acting on the surface

time step in the transient strategy

Cauchy stress tensor

fluid velocity
$<_{-}>$

Derivative of $<_{-}>$with respect to time

$<_{-}>$

$<_{-}>_{\delta}$

$<_{-}>_{\Delta}$

$<_{-}>^{n}$

$<_{-}>^{k}$
Variation of $<_{-}>$

Increment of $<_{-}>$

$<_{-}>$at time $t_{n}$

$<_{-}>$at $k^{\text {th }}$ iteration at time $t_{n+1}$

\section{References}

[1] Sterl A 1990 Numerical simulation of liquid-metal MHD flows in rectangular ducts. J. Fluid Mech. 216: 161-191

[2] Hua T Q and Walker J S 1995 MHD flow in rectangular ducts with inclined non-uniform transverse magnetic field. Fusion Eng. Des. 27: 703-710

[3] Borghi C A, Cristofolini A and Minak G 1996 Numerical methods for the solution of the electrodynamics in magnetohydrodynamic flows. IEEE Trans. Magn. 32(3): 1010-1013

[4] Farahbakhsh I and Ghassemi H 2010 Numerical investigation of the Lorentz force effect on the vortex transfiguration in a two-dimensional lid-driven cavity. Proc. Inst. Mech. Eng. Part C: J. Mech. Eng. Sci. 224(6): 1217-1230

[5] Peterson J S 1988 On the finite element approximation of incompressible flows of an electrically conducting fluid. Numer. Methods Partial Differ. Equ. 4: 57-68

[6] Morley N B , Smolentsev S, Munipalli R, Ni M J, Gao D and Abdou M 2004 Progress on the modeling of liquid metal, free surface, MHD flows for fusion liquid walls. Fusion Eng. Des. 72: 3-34

[7] Shakeri F and Dehghan M 2011 A finite volume spectral element method for solving magnetohydrodynamic (MHD) equations. Appl. Numer. Math. 61: 1-23

[8] Verardi S L L and Cardoso J R 1998 A solution of twodimensional magnetohydrodynamic flow using the finite element method. IEEE Trans. Magn. 34(5): 3134-3137

[9] Planas R, Badia S and Codina R 2011 Approximation of the inductionless MHD problem using a stabilized finite element method. J. Comput. Phys. 230: 2977-2996

[10] Boffi D, Brezzi F and Fortin M 2013 Mixed finite elements and applications. Berlin: Springer

[11] Salah N B, Soulaimani A and Habashi W G 2001 A finite element method for magneto-hydrodynamics. Comput. Methods Appl. Mech. Eng. 190: 5867-5892

[12] Salah N B 1999 A finite element method for the fully coupled magneto-hydrodynamics. PhD Thesis, Concordia University, Montreal, Canada

[13] Salah N B, Soulaimani A, Habashi W G and Fortin M 1999 A conservative stabilized finite element method for the magneto-hydrodynamic equations. Int. J. Numer. Methods Fluids 29: 535-554

[14] Shadid J N, Pawlowski R P, Banks J W, Chacn L, Lin P T and Tuminaro R S 2010 Towards a scalable fully-implicit fully-coupled resistive MHD formulation with stabilized FE methods. J. Comput. Phys. 229: 7649-7671

[15] Nesliturk A I and Tezer-Sezgin M 2006 Finite element method solution of electrically driven magnetohydrodynamic flow. J. Comput. Appl. Math. 192: 339-352 
[16] Guermond J L, Lorat J and Nore C 2003 A new finite element method for magneto-dynamical problems: two-dimensional results. Eur. J. Mech. B Fluids 22: 555-579

[17] Codina R and Silva N H 2006 Stabilized finite element approximation of the stationary magneto-hydrodynamics equations. Comput. Mech. 38: 344-355

[18] Gerbeau J F 2000 A stabilized finite element method for the incompressible magnetohydrodynamic equations. Numer. Math. 87: 83-111

[19] Schotzau D 2004 Mixed finite element methods for stationary incompressible magnetohydrodynamics. Numer. Math. 96: $771-800$

[20] Zhang G, He Y and Yang D 2014 Analysis of coupling iterations based on the finite element method for stationary magnetohydrodynamics on a general domain. Comput. Math. Appl. 68: 770-788

[21] Greif C, Li D, Schtzau D and Wei X 2010 A mixed finite element method with exactly divergence-free velocities for incompressible magnetohydrodynamics. Comput. Methods Appl. Mech. Eng. 199: 2840-2855

[22] Jin D, Ledger P D and Gil A J 2014 An hp-fem framework for the simulation of electrostrictive and magnetostrictive materials. Comput. Struct. 133: 131-148

[23] Jin D, Ledger P D and Gil A J 2016 hp-Finite element solution of coupled stationary magnetohydrodynamics problems including magnetostrictive effects. Comput. Struct. 164: 161-180
[24] Jog C S and Kumar R 2010 Shortcomings of discontinuouspressure finite element methods on a class of transient problems. Int. J. Numer. Methods Fluids 62: 313-326

[25] Davidson P A 1990 An introduction to magnetohydrodynamics. Cambridge: Cambridge University Press

[26] Bardi I, Biro O and Preis K 1991 Finite element scheme for 3D cavities without spurious modes. IEEE Trans. Magn. 27: 4036-4039

[27] Jog C S and Nandy A 2014 Mixed finite elements for electromagnetic analysis. Comput. Math. Appl. 68: 887-902

[28] Nandy A and Jog C S 2016 An amplitude finite element formulation for electromagnetic radiation and scattering. Comput. Math. Appl. 71: 1364-1391

[29] Gupta A 2000 WSMP: Watson sparse matrix package part II-direct solution of general sparse systems. IBM Research Report RC 21888 (98472)

[30] Gupta A 2002 Recent advances in direct methods for solving unsymmetric sparse systems of linear equations. ACM Trans. Math. Softw. 28(3): 301-324

[31] Moreau R 1990 Magnetohydrodynamics. Dordrecht: Springer

[32] Moffatt H K 1983 Magnetic field generation in electrically conducting fluids. Cambridge: Cambridge University Press

[33] Hunt J C R 1965 Magnetohydrodynamic flow in rectangular ducts. J. Fluid Mech. 21(4): 577-590 